\title{
P2P-Based, Multi-Attribute Resource Discovery under Real-World Resources and Queries
}

\author{
H. M. N. DILUM BANDARA and ANURA P. JAYASUMANA, Colorado State University
}

\begin{abstract}
Collaborative peer-to-peer (P2P), grid, and cloud computing rely on resource discovery (RD) solutions to aggregate groups of multi-attribute, dynamic, and distributed resources. However, specific characteristics of real-world resources and queries, and their impact on P2P-based RD, are largely unknown. We analyze the characteristics of resources and queries using data from four real-world systems. These characteristics are then used to qualitatively and quantitatively evaluate the fundamental design choices for P2P-based multi-attribute RD. The datasets exhibit several noteworthy features that affect the performance. For example, compared to uniform queries, real-world queries are relatively easier to resolve using unstructured, superpeer, and single-attribute-dominated query-based structured P2P solutions, as queries mostly specify only a small subset of the available attributes and large ranges of attribute values. However, all the solutions are prone to significant load balancing issues, as the resources and queries are highly skewed and correlated. The implications of our findings for improving RD solutions are also discussed.
\end{abstract}

Categories and Subject Descriptors: C.2.4 [Computer-Communication Networks]: Distributed Systems; C.4 [Performance of Systems]: Performance attributes; H.3.3 [Information Systems]: Information Search and Retrieval

General Terms: Design, Measurement, Performance

Additional Key Words and Phrases: Multi-attribute queries, multi-attribute resources, peer-to-peer, resource discovery, simulation

ACM Reference Format:

Bandara, H. M. N. D. and Jayasumana, A. P. 2015. P2P-based, multi-attribute resource discovery under real-world resources and queries. ACM Trans. Internet Technol. 15, 1, Article 5 (February 2015), 35 pages. DOI : http://dx.doi.org/10.1145/2729139

\section{INTRODUCTION}

Collaborative peer-to-peer (P2P) systems, an emerging class of applications, look for diverse peers that could bring in unique resources and capabilities to a virtual community, thereby empowering it to engage in greater tasks beyond what can be accomplished by individual peers, yet are beneficial to all of them [Bandara and Jayasumana 2012b]. In contrast to conventional P2P systems that focus on pairwise interactions between similar types of resources (e.g., file sharing), future P2P applications will thrive on the interactions among groups of diverse and distributed resources. A collaborative P2P system may consist of a set of voluntary nodes, dedicated nodes from multiple grid/cloud sites, or a combination of both. These systems are expected to share a variety of resources such as processor cycles, storage capacity,

This research is supported in part by the Engineering Research Center program of the National Science Foundation under award number 0313747.

Authors' addresses: H. M. N. D. Bandara and A. P. Jayasumana (corresponding author), Department of Electrical and Computer Engineering, Colorado State University, Fort Collins, CO 80523; email: anura.jayasumana@colostate.edu.

Permission to make digital or hard copies of all or part of this work for personal or classroom use is granted without fee provided that copies are not made or distributed for profit or commercial advantage and that copies bear this notice and the full citation on the first page. Copyrights for components of this work owned by others than ACM must be honored. Abstracting with credit is permitted. To copy otherwise, or republish, to post on servers or to redistribute to lists, requires prior specific permission and/or a fee. Request permissions from permissions@acm.org.

(C) 2015 Copyright held by the Owner/Author. Publication rights licensed to ACM.

$1533-5399 / 2015 / 02-A R T 5 \$ 15.00$

DOI : http://dx.doi.org/10.1145/2729139 
network bandwidth, sensors/actuators, services, middleware, scientific algorithms, and data to not only consume a variety of contents but also to generate, modify, and manage these contents.

To consider the salient features of collaborative P2P systems, we use four representative applications. First is collaborative adaptive sensing of the atmosphere (CASA) [McLaughlin et al. 2009], an emerging network of weather radars that operates collaboratively to detect hazardous atmospheric conditions such as tornados and flash floods. Collaborative P2P data fusion provides an attractive implementation choice for real-time radar data fusion in CASA, wherein multiple data volumes are constantly being generated, processed, and pushed and pulled among radars, storage, and processing nodes [Lee et al. 2012]. Second, cloud computing is transforming the way we host and run applications because of its rapid scalability and pay-as-you-go economic model. Open cloud initiatives are pressing for interoperability among multiple cloud providers and sites. Alternatively, community cloud computing [Briscoe and Marinos 2009], based on underutilized computing resources in homes/businesses, targets issues such as centralized data, privacy, proprietary applications, and cascading failures in modern cloud computing systems. Certain applications also benefit from a mixture of dedicated and voluntary cloud resources [Andrzejak et al. 2010]. Collaborative $\mathrm{P} 2 \mathrm{P}$ systems are useful for interconnecting resources, which may be dedicated or voluntary, in multisite or community cloud systems. We refer to such systems as P2P clouds. The third example is global environment for network innovations (GENI) [Elliott 2009], a collaborative and exploratory platform that allows users to aggregate diverse hardware and software resources from multiple administrative domains for a common task. A collaborative P2P system is a natural fit for GENI because of its distributed, dynamic, and collaborative nature. Fourth, the value of social networks can be enhanced by allowing users to share diverse resources available in their mobile devices. For example, a group sharing their holiday experiences in a coffee shop could use one of their members' projection phone to show pictures from others' mobiles or tablets, or stream videos from their home servers. Such applications, referred to as mobile social networks, also benefit from collaborative P2P technology.

CASA, GENI, P2P clouds, and mobile social networks depend on some form of collaboration among resources. Therefore, these applications need the ability to locate and aggregate groups of complex resources as and when needed to meet the performance and quality-of-service (QoS) requirements. P2P-based resource discovery (RD) is a natural fit for these distributed, collaborative applications and further enhances their scalability, load balancing, and robustness. P2P-based RD solutions have been also proposed for many conventional applications such as grid, desktop grid, and cloud computing [Cai et al. 2004; Lazaro et al. 2013; Shen and Xu 2012b], as timely aggregation of complex and distributed resources is becoming increasingly necessary due to the proliferation of parallel applications that utilize multiple and distributed resources. Yet it is nontrivial to discover and utilize heterogeneous, multi-attribute, and dynamic resources that are distributed. Nevertheless, a good RD solution should satisfy several key phases where they: (1) efficiently advertise all the resources and their state, (2) query to find resources that satisfy application requirements, (3) match resources according to application and resource constraints, and (4) bind resources and applications to ensure guaranteed service [Bandara and Jayasumana 2012b]. Moreover, solutions need to be adaptive, fault tolerant, and should satisfy multiple aspects of scalability such as query resolution latency, number of attributes, number of messages, and routing state. The focus of this article is on the first two phases, namely advertising and querying.

Significant progress has been made in multi-attribute RD in grid computing and P2P [Albrecht et al. 2008; Bharambe et al. 2004; Cai et al. 2004; Costa et al. 2009; 
Lazaro et al. 2013; Shen 2009; Shen and Hwang 2012; Shen and Xu 2012]. However, compared to single-attribute P2P systems such as file sharing, formal characterization of real-world, multi-attribute resources and queries received attention only recently [Bandara and Jayasumana 2011b; Heien et al. 2012]. Characteristics of static attributes (i.e., attribute values are fixed, e.g., CPU speed) of nodes from a set of volunteer computing systems are presented in Heien et al. [2012]. Our preliminary work in Bandara and Jayasumana [2011b] presented both the static and dynamic (i.e., attribute values change with time, e.g., free CPU) resource and query characteristics of PlanetLab [Kim et al. 2011] and SETI@home [Anderson and Fedak 2006] nodes. In the absence of information, data, and understanding of real-life, multi-attribute resource and query characteristics, designs and evaluations of existing RD solutions have relied on many simplifying assumptions. For example, they assume independent and identically distributed (i.i.d.) attributes [Shen and Hwang 2012; Shen and $\mathrm{Xu}$ 2012], uniform, Zipf's, or Pareto distribution of all the resources/queries [Costa et al. 2009; Shen et al. 2013; Shen and Hwang 2012; Shen and Xu 2012], and queries specifying a large number of attributes and a small range of attribute values [Cai et al. 2004; Shen and Xu 2012; Shen et al. 2013]. Moreover, the cost of updating dynamic attributes is ignored in all designs. Such assumptions affect both the designs and performance analyses of RD solutions, and consequently their applicability under real workloads [Bandara and Jayasumana 2012a]. Furthermore, direct comparison of these solutions is impractical due to the diversity of designs. Therefore, a formal and detailed comparison of fundamental design choices for P2P-based RD with respect to the behavior learned from actual systems is needed. Such an analysis will not only provide a better understanding of the applicability of different design choices but also help identify best practices for resource aggregation in emerging collaborative P2P systems.

We qualitatively and quantitatively evaluate the fundamental design choices for P2P-based, multi-attribute RD using the characteristics learned from real-world systems. Qualitative evaluation considers aspects such as load balancing, support for new attributes, and freshness of query results under the learned characteristics. Quantitative evaluation uses performance bounds and simulation results (using real datasets) to evaluate aspects such as cost of $\mathrm{RD}$, load distribution, routing state, and scalability. The main contributions of this article are as follows.

- We give an approximation to capture the overall cost of RD in terms of overlay messages. It helps identify important parameters that affect the cost of $\mathrm{RD}$ and performance bounds.

— Characteristics of multi-attribute resources from PlanetLab, SETI@home, EGI grid, and a campus network are then analyzed to determine the nature of parameters (e.g., their distributions and temporal characteristics) that affect the cost of RD in different systems. PlanetLab data are also used to analyze the multi-attribute query characteristics. Such an understanding is useful in designing and evaluating RD solutions and job schedulers.

- A representative subset of design choices, based on centralized, unstructured, superpeer, and structured $\mathrm{P} 2 \mathrm{P}$ architectures, is then qualitatively and quantitatively evaluated using the learned characteristics. Nine design choices are evaluated based on the cost of advertising and querying resources, routing-table size, load balancing, and scalability. A simulation-based study using node and query traces from PlanetLab is used for the quantitative evaluation. These findings help identify best practices for resource aggregation.

- The implications of real-world resource and query characteristics for improving and developing new P2P-based RD solutions are discussed. 
Our findings include the following.

- Attributes of most resources are highly skewed and correlated. Attribute values have different marginal distributions and most of them are too complex/skewed to be described using any of the standard probability distributions. Ones that fit a known distribution satisfy Gaussian, generalized extreme value, and generalized Pareto distributions. While resources are characterized by many attributes, most attributes have only a few distinct sets of values. Attribute values change at different rates and a few attributes change quite rapidly.

- Attribute values specified in queries are skewed; however, they do not satisfy a Zipf's-like distribution. Queries are less specific where each query tends to specify only a small subset of the available attributes and large ranges of attribute values.

- Simulation-based analysis indicates that real-world queries are relatively easier to resolve using unstructured, superpeer, and single-attribute-dominated query-based structured P2P architectures compared to uniform queries used in conventional studies. The cost of $\mathrm{RD}$ in ring-based structured $\mathrm{P} 2 \mathrm{P}$ systems is effectively $O(n)$, where $n$ is the number of nodes in the overlay, as most range queries specify large ranges of attribute values. The cost of advertising dynamic attributes is significant and increases with the number of attributes, and hence should not be ignored in performance studies. Furthermore, all the design choices are prone to significant load balancing issues where only a few nodes are mainly involved in answering the majority of queries and indexing resources. Therefore, existing design choices perform poorly under realistic workloads.

Our work differs from Heien et al. [2012] in several aspects. Heien et al. analyzed the evolution of static attributes of multiple volunteer computing systems (e.g., SETI@home, Einstein@home, and World Community Grid) over five years and developed a model to forecast how the composition of hardware resources will evolve in the future. Conversely, we analyze both the static and dynamic attributes as well as shortterm (ranging from a few minutes to days) changes in dynamic attributes. We also consider four different computing environments. Multi-attribute query characteristics are also analyzed using PlanetLab data. Several other attempts to model grid computing resources are presented in Kee et al. [2004], Lu and Dinda [2003], and Sulistio et al. [2008]; however, they also do not capture the dynamic attributes. While Shen and $\mathrm{Xu}$ [2012] analyzed the performance of several distributed hash-table (DHT)-based designs, they extensively relied on conventional assumptions. Consequently, the authors concluded that the query cost increases sublinearly with the number of nodes (i.e., $\log n$ ) and linearly with the number of attributes. Moreover, they concluded that LORM could provide better index size distribution than other DHT-based solutions. Conversely, our analysis based on real-world resources and queries shows that query cost is $O(n)$ and reduces with the number of attributes (in certain DHT-based designs). Moreover, index size and query load cannot be balanced using conventional DHT-based techniques due to highly skewed and correlated attributes. Furthermore, our analysis also considers other fundamental design choices, including centralized, unstructured, and superpeer architectures, as well as the dynamic attributes. Our work not only demonstrates that real-world, multi-attribute resource and query characteristics diverge substantially from conventional assumptions, but also highlights the need for understanding the characteristics of different systems for the correct design, validation, and performance analysis of RD solutions and job schedulers. Our analysis of PlanetLab resources and queries is complementary to the work in Kim et al. [2011], which analyzes the behavior of workloads in PlanetLab.

Section 2 presents an approximation for the cost of RD. Four datasets are described in Section 3 and their characteristics analyzed in Section 4. Different resource 
advertising and querying options are qualitatively and quantitatively evaluated in Section 5. Section 6 presents the simulation setup and performance analysis. Implications and best practices for future RD solutions are discussed in Section 7. Concluding remarks are presented in Section 8. This is an extended version of the papers in Bandara and Jayasumana [2011b, 2012a]. The major extensions include the analysis of more recent and multiple datasets, extending the RD cost equation for different design choices for P2P-based RD, extended performance analysis, and a detailed discussion on the implications of our findings for improving P2P-based RD.

\section{COST OF ADVERTISING AND QUERYING RESOURCES}

We consider those resources that are characterized by multiple attributes, for example, a computing node is characterized by its CPU speed, memory, operating system, etc. Similar to advertising file names in file sharing, these attributes are advertised to a central database, neighboring nodes, or nodes implementing DHTs. All the attributes of a resource may be advertised together as a vector, or separately as a set of scalar values. We analyze the cost of RD in terms of the number of overlay messages involved in advertising such resources and querying them. Our objective is to derive a firstorder approximation to capture the cost of RD. This serves two purposes. First, it helps to identify a set of key parameters and their relationship to the cost of advertising and querying (in Section 4, we identify how these parameters behave in real-world systems). Second, it helps to identify the performance bounds of RD solutions under real-world resources and queries. This is presented in Section 5, where the analysis is extended to represent the cost of RD in centralized, unstructured, and structured P2Pbased architectures using the real-world resource and query characteristics learned in Section 4. These performance bounds are then validated in Section 6 using a set of simulations.

Consider a P2P overlay used to index (i.e., keep track of) resources, where resources are advertised to a distributed set of nodes, and queries are resolved by contacting these nodes. Let $\mathbf{R}$ be the set of resources in the system and $\mathbf{A}$ the set of attributes used to describe those resources. We use boldface symbols to refer to a set and the corresponding italic symbol refers to its cardinality, for instance, $R=|\mathbf{R}|$. A list of symbols is given in Table I. Each resource $r \in \mathbf{R}$ is defined as follows [Bandara and Jayasumana 2012b]:

$$
r=\left(a_{1}=v_{1}, a_{2}=v_{2}, \ldots, a_{i}=v_{i}\right) .
$$

Each attribute $a_{i} \in \mathbf{A}$ has a corresponding value $v_{i} \in \mathbf{D}_{\mathbf{i}}$ that belongs to a given domain $\mathbf{D}_{\mathbf{i}}$. The $\mathbf{D}_{\mathbf{i}}$ 's are typically bounded, may be continuous or discrete, or correspond to a set of categories/names. For example, free CPU is continuous, number of CPU cores is discrete (integer), operating system is a category, and a file is represented by a name. A multi-attribute Resource Specification (RS) with such a set of attributes may look like the following:

$$
R S=\left(\begin{array}{l}
\text { CPUSpeed }=2.4 \mathrm{GHz}, \text { NumCores }=2, \text { CPUFree }=53 \%, \\
\text { MemoryFree }=1071 \mathrm{MB}, \mathrm{OS}=\text { "Linux_2.6.31" }
\end{array}\right) .
$$

Attribute values are classified as static (e.g., CPU speed, total memory, and operating system) or dynamic (e.g., free CPU, memory, and bandwidth). Let $\mathbf{a}_{\mathbf{r}} \subseteq \mathbf{A}$ be the collection of $a_{i}$ 's that is used to describe $r$. Let $\mathbf{A}^{\mathbf{s}}$ and $\mathbf{A}^{\mathbf{d}}$ be the set of static and dynamic attributes in the system, respectively. $\mathbf{A}=\mathbf{A}^{\mathbf{s}} \cup \mathbf{A}^{\mathbf{d}}$. Similarly, let $\mathbf{a}_{\mathbf{r}}^{\mathbf{s}}$ and $\mathbf{a}_{\mathbf{r}}^{\mathbf{d}}$ be the subsets of static and dynamic attributes of $\mathbf{a}_{\mathbf{r}}$. Dynamic attributes need to be re-advertised whenever their values/states change. Let $\lambda_{r}^{i}$ be the rate at which the change in $i$-th attribute of $\mathbf{a}_{\mathbf{r}}$ occurs. The cost of advertising a dynamic attribute is proportional to 
Table I. List of Symbols

\begin{tabular}{|l|l|}
\hline \multicolumn{1}{|c|}{ Symbol } & \multicolumn{1}{c|}{ Name } \\
\hline $\mathbf{a}_{\mathbf{r}}$ & Attribute set of resource $r$ \\
\hline $\mathbf{a}_{\mathbf{r}}^{\mathbf{S}} / \mathbf{a}_{\mathbf{r}}^{\mathbf{d}}$ & Set of static/dynamic attributes of resource $r$ \\
\hline $\mathbf{a}_{\mathbf{q}}$ & Attribute set of query $q$ \\
\hline $\mathbf{A} / A$ & Set/number of distinct attributes in system \\
\hline $\mathbf{A}^{\mathbf{S}} / \mathbf{A}^{\mathbf{d}}$ & Set of static/dynamic attributes in system \\
\hline$c_{r}^{i}$ & Number of copies of $i$-th attribute of resource $r$ \\
\hline $\mathbf{D}_{\mathbf{i}}$ & Domain of $i$-th attribute \\
\hline$h_{\text {Advertise }}^{i} / h_{\text {Query }}^{i}$ & Hops to advertise/query $i$-th attribute \\
\hline$h_{T T L}$ & Time to live value used by random walks \\
\hline$l_{i}$ & Lower bound of $i$-th attribute \\
\hline$m_{q}$ & Desired number of resources in query $q$ \\
\hline$n$ & Number of nodes in system \\
\hline$Q(t)$ & Set of queries issued in time interval $t$ \\
\hline $\mathbf{R} / R$ & Set/number of resources in system \\
\hline$t$ & Time \\
\hline$u_{i}$ & Upper bound of $i$-th attribute \\
\hline$v_{i}$ & Value of $i$-th attribute \\
\hline$\lambda_{r}^{i}$ & Rate of change of $i$-th attribute of resource $r$ \\
\hline
\end{tabular}

its rate of change. However, in practice, $\lambda_{r}^{i}$ is bounded by the sampling rate. For example, though CPUFree changes frequently, the applications sample them infrequently (every few seconds or minutes). In some cases, multiple copies of $a_{i}$ are placed in different databases, neighboring nodes, or DHTs. Let $c_{r}^{i}$ be the number of copies of the $i$-th attribute of $\mathbf{a}_{\mathbf{r}}$.

We are interested in the number of messages that are either sent or forwarded within the overlay to advertise and query resources. Cost of acknowledgement messages is ignored. Typically, the time required to resolve a query within a node is lower than the inter-node communication time. Therefore, if each overlay link is assumed to have approximately the same latency, then the query resolution latency can be also represented using the number of overlay hops. Assuming each attribute $a_{i}$ is advertised separately, the total cost of advertising $r$ is

$$
C_{\text {Advertise }}^{r}=\sum_{i \in \mathbf{a}_{\mathbf{r}}} c_{r}^{i} h_{\text {Advertise }}^{i}+\sum_{j \in \mathbf{a}_{\mathbf{r}}} c_{r}^{j} h_{\text {Advertise }}^{j} \lambda_{r}^{j} t=\sum_{i \in \mathbf{a}_{\mathbf{r}}} c_{r}^{i} h_{\text {Advertise }}^{i}\left(1+\lambda_{r}^{i} t\right),
$$

where $h_{\text {Advertise }}^{i}$ is the number of overlay hops that the advertising message corresponding to the $i$-th attribute travels. First summation captures the initial cost of advertising all the attributes. Second summation captures the recurrent cost of advertising dynamic attributes within time $t$. Resources need to re-enter the system after churn or failure. Moreover, resources indexed in unstructured P2P systems and DHTs typically expire after a predefined timeout. In either case, resource attributes need to be re-advertised. Therefore, even the static attributes can be interpreted as behaving like dynamic attributes, but with much lower turnover. Hence, $\lambda_{r}^{i}$ should be defined considering the rate of change of attributes, sampling rate, and both the available and unavailable time of $r . \lambda_{r}^{i}$ is lower for static attributes compared to that for dynamic attributes.

Let $\mathbf{Q}(\boldsymbol{t})$ be the set of all queries issued within the time interval $t$. A multi-attribute range query $q \in \mathbf{Q}(\boldsymbol{t})$ is defined as follows [Bandara and Jayasumana 2012b]:

$$
q=\left(m_{q}, a_{1} \in\left[l_{1}, u_{1}\right], a_{2} \in\left[l_{2}, u_{2}\right], \ldots, a_{i} \in\left[l_{i}, u_{i}\right]\right)
$$


where, $m_{q} \in \mathbf{Z}^{+}$specifies the required number of resources and $a_{i} \in\left[l_{i}, u_{i}\right]$ specifies the desired range of attribute values $\left(l_{i}\right.$ and $u_{i}$ are lower and upper bounds, respectively). A query that requests for five computing nodes with a given CPU speed, free memory, and operating system may look like the following:

$$
q=\left(\begin{array}{l}
m_{q}=5, \text { CPUSpeed } \in[2.0 \mathrm{GHz}, \mathrm{MAX}], \\
\text { MemoryFree } \in[256 \mathrm{MB}, 512 \mathrm{MB}], \text { OS = "Linux_2.6.32" }
\end{array}\right),
$$

where $M A X_{i}$ is the upper bound of the domain $\mathbf{D}_{\mathbf{i}}$. The set of attributes specified in a query $\left(\mathbf{a}_{\mathbf{q}}\right)$ may contain only a subset of the attributes that are used to describe resources (i.e., $\mathbf{a}_{\mathbf{q}} \subseteq \mathbf{A}$ ). When $l_{i}=u_{i}, a_{i} \in \mathbf{a}_{\mathbf{q}}, q$ is referred to as a point query. In practice, attributes in a query may specify a mixture of point and range values. Unspecified attributes are considered as “don't care". Query $q$ is referred to as a single-attribute query when $a_{q}=1$ and a multi-attribute query when $a_{q}>1$. In practice, it may be necessary to find/discover more than $m_{q}$ resources, as some of the selected resources may not be available by the time they are required for use, due to resource churn/failure. Moreover, $\mathrm{RD}$ systems may not index all the attributes used to describe a resource, for example, only the static attributes are indexed in Costa et al. [2009]. In such cases, unadvertised attributes have to be validated by directly contacting the resources. Contacting the resource is also important when a binding has to be established between the resource and the application planning to use it [Bandara and Jayasumana 2012b]. Let $f\left(m_{q}, \mathbf{R}, \mathbf{A}_{\text {Index }}\right)$ indicate the cost associated with these complex scenarios. $\mathbf{A}_{\text {Index }} \subseteq \mathbf{A}$ is the subset of attributes indexed in the P2P overlay. Then the cost of a point query can be given as

$$
C_{\text {Query-Point }}^{q}=\sum_{i \in \mathbf{a}_{\mathbf{q}}} h_{\text {Query }}^{i}+f\left(m_{q}, \mathbf{R}, \mathrm{A}_{\mathbf{I n d e x}}\right),
$$

where $h_{\text {Query }}^{i}$ is the number of overlay hops required to reach the node that indexes the value of the $i$-th attribute defined in $q$, and $f($.) captures the cost of directly contacting resources to either validate those attributes that are not advertised or to establish a binding between a resource and the application. After reaching the first node, queries that specify a range of attribute values need to be forwarded further. As in Albrecht et al. [2008], Cai et al. [2004], and Shen and Xu [2012], we initially assume the range of attribute values $\left[l_{i}, u_{i}\right]$ to be uniformly distributed within the overlay. As the domain of attribute $i\left(D_{i}\right)$ is mapped to $n$ nodes, each node is responsible for indexing $D_{i} / n$ fraction of the attribute space. Therefore, a range query that searches for $\left[l_{i}, u_{i}\right]$ has to go through $\left(u_{i}-l_{i}\right) n / D_{i}$ nodes (i.e., range to search divided by range per node). Then (4) can be extended to indicate the overall cost of a range query as follows:

$$
C_{\text {Query }}^{q}=\sum_{i \in \mathbf{a}_{\mathbf{q}}}\left(h_{\text {Query }}^{i}+\left\lceil\frac{\left(u_{i}-l_{i}\right)}{D_{i}} n\right\rceil-1\right)+f\left(m_{q}, \mathbf{R}, \mathbf{A}_{\text {Index }}\right) .
$$

Then the overall cost of $\mathrm{RD}$ is

$$
C_{\text {Total }}=\sum_{r \in \mathbf{R}} C_{\text {Advertise }}^{r}+\sum_{q \in \mathbf{Q}(\mathbf{t})} C_{\text {Query }}^{q} .
$$

\section{RESOURCE AND QUERY DATASETS}

We use four datasets from PlanetLab, SETI@home, EGI grid, and our campus to identify the nature of parameters that affect the cost of RD. The set of resource attributes that are used in the analysis are described first. Then details on traces used in this study are presented. 


\subsection{Node Model}

Though resources are characterized by many static, dynamic, and categorical attributes, only a subset of them are useful in RD. Even among these attributes, some are more important than others. Therefore, we consider 11 representative attributes that are essential to characterize a typical node useful for collaborative P2P, grid, and cloud computing systems.

(1) CPUSpeed. This is the processor clock speed in GHz. It provides insight on the relative computing power of a node.

(2) CPUArchi. This is the CPU architecture, such as $\times 86$ and SPARC.

(3) NumCores. It is the number of processor cores of a node and indicates how much parallelism in processing is possible. Some processors may count hardware-level threads as separate cores.

(4) CPUFree. (100 - percentage CPU utilization). This indicates to what extent the $\mathrm{CPU}(\mathrm{s})$ is available for processing. When multiple cores are available, the average value is given.

(5) CPULoad. It is the number of active processes competing or waiting for the CPU. It is typically represented as one min (1MinLoad), five min (5MinLoad), and 15 min (15MinLoad) exponentially weighted moving averages of the number of competing processes. CPULoad indicates how long a user process has to wait for the CPU. Both CPUFree and CPULoad are complementary to each other, as a large CPULoad does not necessarily mean high CPU utilization (e.g., processes could be blocked for $\mathrm{I} / \mathrm{O}$ ).

(6) MemSize. This is the size of volatile memory in GB.

(7) MemFree. It is free user-level memory as a percentage or in GB and indicates how much memory is available for user processes.

(8) DiskFree. This is the free disk space in GB.

(9) TxRate. This indicates the average transmission rate in Kbps. In conjunction with the bandwidth limit specified by most nodes, it provides insight on available bandwidth. It may also give insight on how much bandwidth will be available if an application uses the node exclusively.

(10) RxRate. This denotes the average receive rate in Kbps.

(11) OS. It is the type of operating system and version.

CPUSpeed, NumCores, and MemSize are static, CPUArchi and OS are categorical, and the rest of the attributes are dynamic. RD solutions and job scheduling algorithms for latency-sensitive applications are typically interested only in short-term trends. Therefore, we capture statistical characteristics that are valid for several minutes to a few weeks.

\subsection{PlanetLab}

PlanetLab [Kim et al. 2011; Park and Pai 2006] is a global research network that supports the development of new network services. It provides a versatile platform for testing distributed applications and protocols by aggregating a globally distributed set of nodes. PlanetLab reflects many characteristics of Internet-based distributed systems such as heterogeneity, multiple end-users, dynamic nodes, and global presence. Hence, it is being used to evaluate many preliminary $\mathrm{P} 2 \mathrm{P}$ protocols and applications. Typical P2P applications include file sharing, streaming, resource sharing, publishersubscriber, and security. We analyzed data from two PlanetLab tools, CoMon [Kim et al. 2011; Park and Pai 2006] and SWORD [Albrecht et al. 2008], to determine multiattribute resource and query characteristics. CoMon is a node and slice monitoring system that provides a snapshot of static and dynamic attributes of all the nodes 
Table II. Summary of Traces

\begin{tabular}{|c|c|c|c|c|c|}
\hline & CoMon & SWORD & SETI@home & GCO & CSU \\
\hline Applications & \multicolumn{2}{|c|}{$\begin{array}{l}\text { Experiments on protocols \& } \\
\text { applications, e.g., P2P file \& } \\
\text { resource sharing, streaming, \& } \\
\text { security }\end{array}$} & $\begin{array}{l}\text { Volunteer } \\
\text { computing, } \\
\text { e.g., } \\
\text { astronomy } \\
\text { research }\end{array}$ & $\begin{array}{l}\text { Grid } \\
\text { computing, } \\
\text { e.g., } \\
\text { high-energy } \\
\text { physics }\end{array}$ & $\begin{array}{l}\text { Campus } \\
\text { computing, } \\
\text { e.g., } \\
\text { teaching } \\
\text { \& research }\end{array}$ \\
\hline $\begin{array}{l}\text { Resource/query } \\
\text { representation }\end{array}$ & As Eq. (1) & As Eq. (3) & As Eq. (1) & As Eq. (1) & As Eq. (1) \\
\hline $\begin{array}{l}\text { No of } \\
\text { nodes/queries }\end{array}$ & $\sim 650$ & 915 & 234,421 & 205 & 387 \\
\hline No of attributes & 64 & 49 & 34 & 87 & 27 \\
\hline $\begin{array}{l}\text { Attributes useful } \\
\text { in RD }\end{array}$ & 46 & 49 & 25 & 17 & 17 \\
\hline Static attributes & 12 & 8 & 21 & 6 & 8 \\
\hline $\begin{array}{l}\text { Dynamic } \\
\text { attributes }\end{array}$ & 34 & 41 & 4 & 11 & 9 \\
\hline Sampling interval & $5 \min$ & Not tracked & $\begin{array}{l}\text { Hours to } \\
\text { days }\end{array}$ & $1 \mathrm{~min}$ & $1 \mathrm{~min}$ \\
\hline Date(s) & $2011 / 2 / 1-14$ & 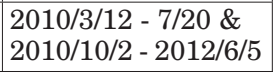 & $\begin{array}{l}\text { Active as of } \\
2012 / 4 / 30\end{array}$ & $2012 / 4 / 23-5 / 6$ & $2011 / 12 / 1-14$ \\
\hline
\end{tabular}

every five minutes. Table II summarizes the traces used in this study. Further details on specific attributes of all four systems are given in Bandara [2012]. SWORD is a multi-attribute RD tool for PlanetLab. It enables users to query for multiple groups of nodes while specifying inter- and intra-group latency and bandwidth constraints. Though initially designed as a $\mathrm{P} 2 \mathrm{P}$ solution, its current deployment is centralized and does not support latency and bandwidth constraints due lack of such data from PlanetLab. This dataset provides a unique insight into real-world, multi-attribute, and multiresource queries that are unknown, albeit the analysis is based on 915 queries (due to light usage).

\subsection{SETI@home}

BOINC [Anderson and Reed 2009] is a volunteer computing platform (a.k.a. desktop grid) that is used to remotely execute jobs using idle computing resources. SETI@home is one of the largest BOINC deployments and utilizes a much larger and diverse set of nodes compared to other datasets. Detailed representation of attribute values of SETI nodes is presented in Anderson and Fedak [2006] and Heien et al. [2012] but their characteristics are not analyzed in the context of RD systems. Therefore, we briefly analyze the distribution of resource attributes collected from SETI@home [Anderson and Reed 2009]. Except for DiskFree, BOINC collects only the static attributes of a node. Attribute values are collected only when a node contacts a BOINC server, therefore the time between two samples varies from several hours to a few days. Hence, DiskFree is not sampled periodically. BOINC also collects CPU performance in terms of Dhrystone (integer) and Whetstone (floating-point) benchmarks. BOINC is a pullbased system where nodes request jobs based on their hardware resources and hence do not support a resource query mechanism.

\subsection{EGI Grid}

European grid infrastructure (EGI) aggregates multiple grid computing sites across Europe [Newhouse 2011]. Data related to job submission, workloads, and computing and storage nodes from EGI sites are collected and published through the Grid Observatory [Germain-Renaud et al. 2011a]. We use node traces from green computing 


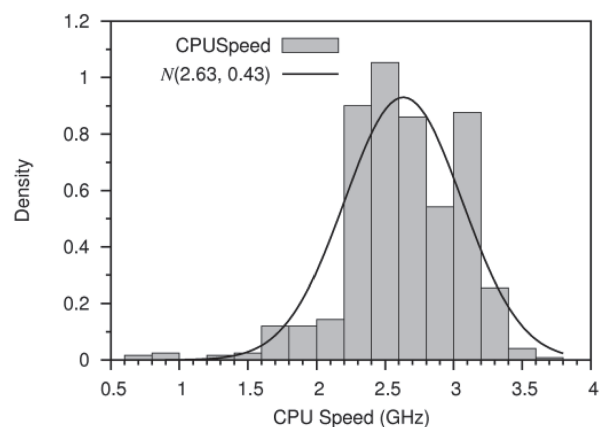

(a)

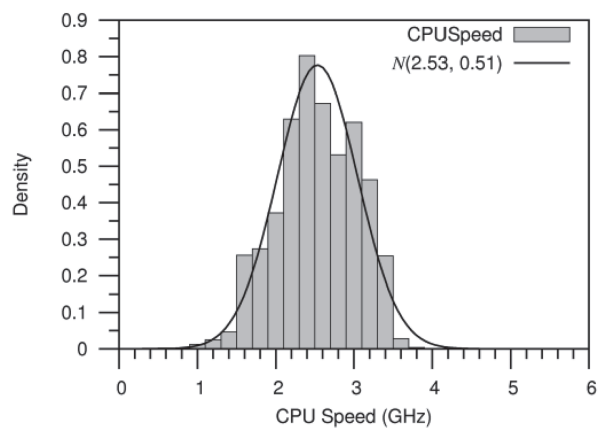

(b)

Fig. 1. Distribution of CPUSpeed: (a) PlanetLab; (b) SETI@home.

observatory (GCO) [Germain-Renaud et al. 2011b], a recently launched subproject within the Grid Observatory, that currently collects static and dynamic attributes of $\sim 200$ computing nodes every minute. Though the number of nodes in the dataset is much smaller than the size of the EGI grid, GCO dataset provides a unique view of grid computing resources due to its high-resolution sampling in both time and number of attributes, which are useful in multi-attribute RD. The specific queries that may have been used to select those nodes for processing are not available.

\subsection{Campus Dataset}

We also collected a dataset within our campus, Colorado State University (CSU). A set of PCs and servers within the Department of Computer Science and another set on the College of Engineering network were sampled every one minute. The dataset includes both Linux and Windows nodes and reflects an enterprise computing environment. Resource characteristics of such environments are becoming important as we are seeing the emergence of applications such as P2P clouds that aggregate residual computing resources from enterprise environments and homes [Briscoe and Marinos 2009]. No explicit query mechanism is provided and users are free to use any unoccupied PC in a lab. Server CPULoads are published in real time on the web, enabling students and researchers to pick unloaded servers manually.

\section{RESOURCE AND QUERY CHARACTERISTICS}

We now describe the characteristics of resources and queries, and the observed behavior of parameters identified in Section 2. The discussion that follows is primarily based on the characteristics of PlanetLab, as we have both the resource and query datasets. Specific characteristics of other datasets are presented to illustrate the commonalities and differences across different systems. A detailed discussion on these datasets and characteristics is available in Bandara [2012].

\subsection{Resource Characteristics}

Figure 1 shows the distribution of CPUSpeed of PlanetLab and SETI@home nodes. Both distributions can be approximated using Gaussian distributions. Due to many identical nodes, CPUSpeed of GCO and CSU datasets were highly skewed and did not fit a standard distribution. Specifically, 11\% of the PlanetLab nodes had identical static attributes such as CPUSpeed, NumCores, MemSize, and DiskSize. This may have been caused by a donation of a set of similar machines to multiple sites. Most sites also had few identical hosts. This is the case even in grid, cloud, and enterprise computing environments where sites may simultaneously deploy or upgrade to a 


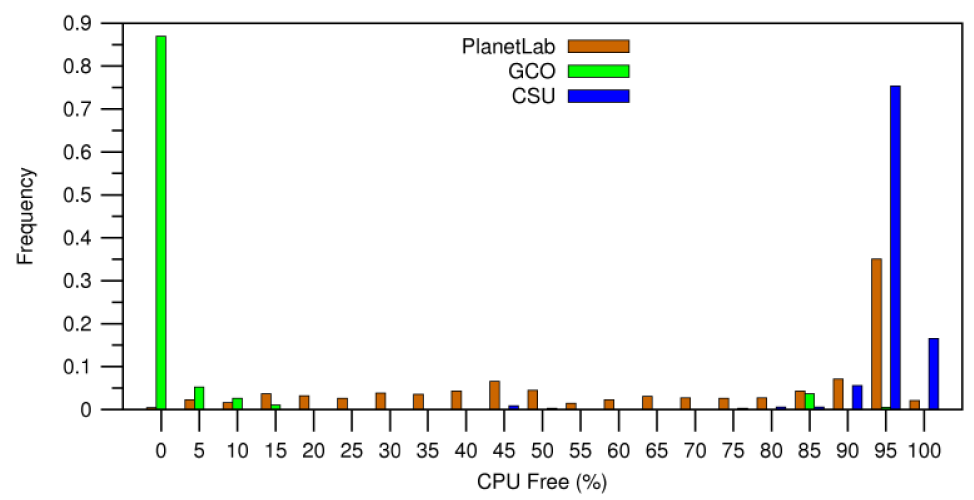

(a)

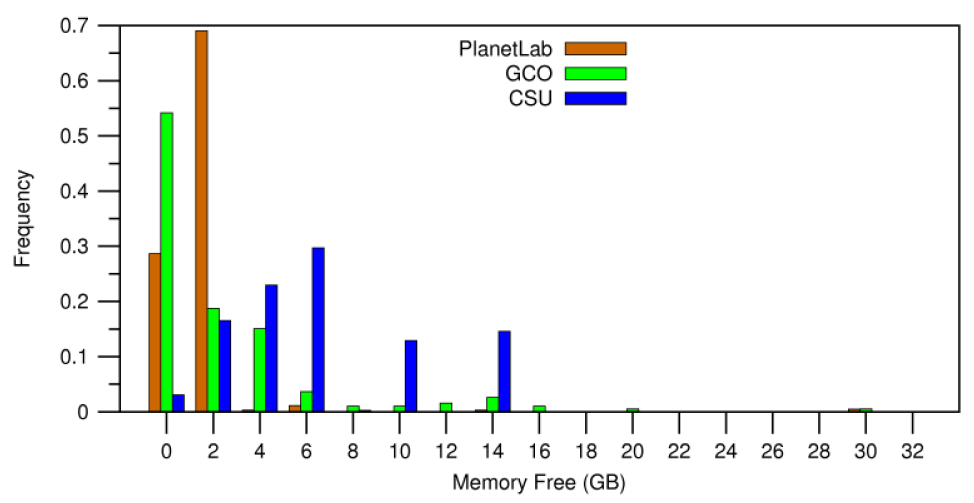

(b)

Fig. 2. Distribution of dynamic attributes during peak times: (a) CPUFree; (b) MemFree.

similar set of machines. For example, the largest set of identical nodes in CSU and GCO datasets corresponded to $14 \%$ and $95 \%$ of the nodes, respectively. Nodes in student labs are typically homogeneous; hence, the four largest sets of identical nodes corresponded to $45 \%$ of the nodes in the CSU dataset. Due to such large sets of homogeneous nodes and the discrete nature of attribute values, NumCores and MemSize of nodes in all the systems do not fit standard distributions. One may argue that we can still attempt to fit data to a set of well-known distributions by applying more complex subsampling techniques, rounding attribute values (e.g., rounding MemSize to the nearest power of two), or discarding some values (e.g., discarding NumCores values that are not powers of two). While we do discard outliers, other techniques are of little value as our objective is to understand the true distributions and behavior of attributes but not to generate synthetic data using them. Such techniques are applied in Heien et al. [2012] for SETI nodes, which are more heterogeneous, as the authors' objective was to generate synthetic data. However, these techniques do not apply well to PlanetLab, GCO, and CSU datasets, as they consist of multiple sets of homogeneous nodes. If the objective is to generate synthetic data, in Bandara and Jayasumana [2011a] we demonstrate that it is more accurate to summarize the data using tables of empirical cumulative distributions (ECDF) and then use empirical copulas to generate synthetic data, as copulas preserve the complex multivariate distributions and correlations among attributes. 
Table III. Distribution of Attribute Values

\begin{tabular}{|l|l|l|}
\hline Distribution of Attribute Values & \multicolumn{1}{|c|}{ PlanetLab* } & \multicolumn{1}{|c|}{ SETI@home* } \\
\hline CPUSpeed $(\mathrm{GHz})$ & N(2.63,0.43) & N(2.53,0.51) \\
\hline DiskFree $(\mathrm{GB})$ & No fit & $G P D(0,104.9,0.51)$ \\
\hline 1MinLoad & $G P D(0,6.82,0.38)$ & N/A \\
\hline TxRate $(\mathrm{Kbps})$ & $G E V(0.51,0.57,0.54)$ & $G E V(12.9,9.8,0.34)$ \\
\hline RxRate $(\mathrm{Kbps})$ & $G E V(0.49,0.54,0.54)$ & $G E V(111.4,122.8,0.84)$ \\
\hline Integer performance (MIPS) & N/A & Weibull $(6,498,2.2)$ \\
\hline Floating-point performance (MIPS) & N/A & Weibull $(2,507,3.4)$ \\
\hline
\end{tabular}

* GEV $(\mu, \sigma, k), \operatorname{GPD}(\mu, \sigma, k), N\left(\mu, \sigma^{2}\right), \operatorname{Weibull}(\lambda, k)$, N/A - Not applicable

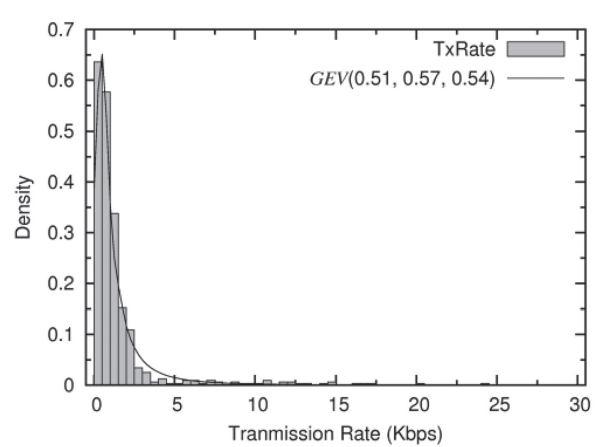

(a)

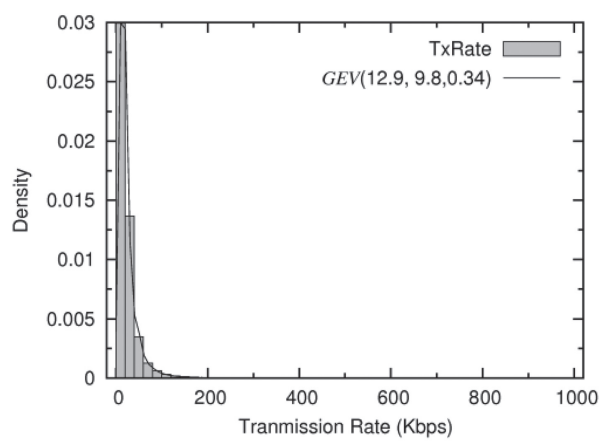

(b)

Fig. 3. Distribution of TxRate: (a) PlanetLab; (b) SETI@home. For fitting the curve for SETI@home, only those nodes with bandwidth utilization up to $1 \mathrm{Mbps}$ are considered.

It was observed that both PlanetLab and GCO (as a system) experience idle and busy periods. Average resource utilization of GCO nodes was higher (lower average CPUFree and MemFree) than the other two systems. For example, Figure 2 illustrates the distribution of CPUFree and MemFree during the peak time, of each system. Large fractions of PlanetLab and CSU nodes were idle (high CPUFree) even during the peak time, whereas GCO nodes were heavily utilized throughout the day. For example, 37\% and $71 \%$ of the PlanetLab nodes had over $95 \%$ of CPUFree and 2GB of MemFree, respectively. Similarly, $92 \%$ and $97 \%$ of the CSU nodes had the same amounts of CPUFree and MemFree. Even though students used the nodes during peak hours, their workloads do not fully utilize the capabilities of the nodes. However, only $0.5 \%$ and $46 \%$ of the GCO nodes had similar amounts of CPUFree and MemFree. Due to the highly skewed distributions, instantaneous values (at a given time $t$ ) of dynamic attributes do not fit standard distributions. Except for SETI nodes, DiskFree also had a similar behavior in the other three datasets. SETI nodes had a skewed but monotonically decreasing distribution that fit the generalized Pareto distribution (GPD). Attributes that satisfy known probability distributions are listed in Table III.

Figure 3 shows the distribution of TxRate of PlanetLab and SETI nodes which can be approximated using the generalized extreme value (GEV) distribution. The RxRate of these nodes can be also approximated using the GEV distribution. The average TxRate of SETI nodes is an order of magnitude lower than the average RxRate (see Table III). This may be a consequence of the asymmetric bandwidth availability in volunteer nodes. The TxRate and RxRate of GCO nodes were skewed and did not fit standard distributions. While it was possible to approximate the distribution of 1MinLoad using GPD, other datasets and 5MinLoad and 15MinLoad did not fit standard distributions. 


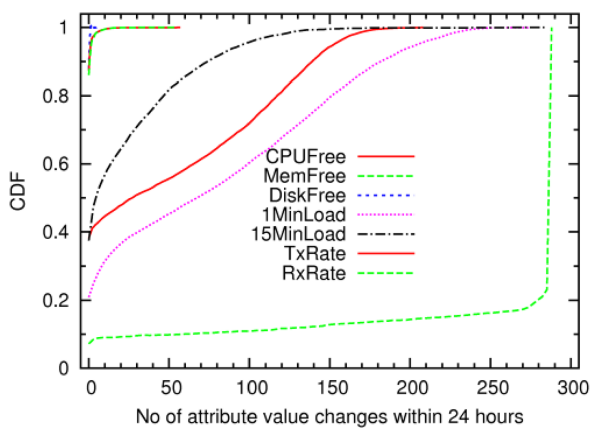

(a)

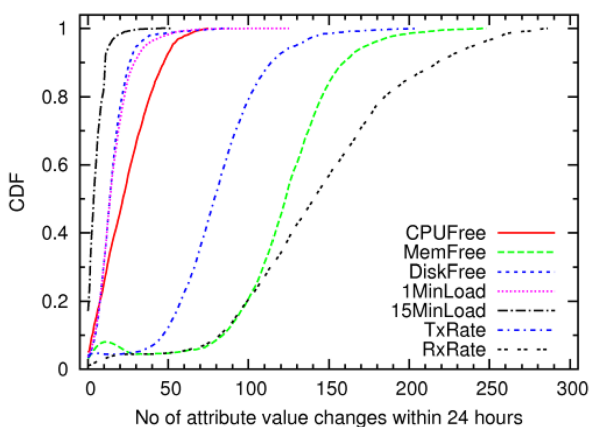

(b)

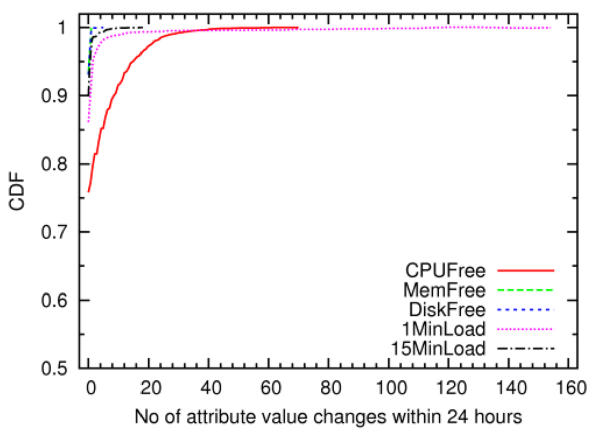

(c)

Fig. 4. Cumulative distribution of number of attribute value changes within 24 hours: (a) PlanetLab; (b) GCO; (c) CSU. Thresholds: CPUFree $= \pm 10 \%$, MemFree $= \pm 100 \mathrm{MB}$, DiskFree $= \pm 5 \mathrm{~GB}$, 1MinLoad $=$ 15MinLoad $= \pm 2$, TxRate $=$ RxRate $= \pm 10 \mathrm{Kbps}$.

It was also observed that integer and floating-point performance of SETI nodes could be approximated by a Weibull distribution. We further observed a few SETI nodes with very large NumCores, MemSize, DiskSize, TxRate, and RxRate. However, these nodes were active only for a few weeks. These could be high-performance machines that utilize SETI as a workload to test and measure their performance. Nevertheless, these nodes provide a significant amount of computing, storage, and networking resources for a short time span.

Distributions of categorical attributes were extremely skewed and had only a few distinct attribute values. For example, CPUArchi of all the PlanetLab, GCO, and CSU nodes and $99 \%$ of the SETI nodes were $\times 86$. All PlanetLab and GCO nodes were Linux but nodes had several different kernel versions. Specifically, $76 \%$ of the CSU nodes ran a Linux variant with different kernel versions, and $82.2 \%$ of the SETI nodes had a Windows variant (44\% of them were Windows 7 ), and $10.6 \%$ and $7 \%$ of the nodes had variants of MacOS and Linux, respectively. The remaining $0.2 \%$ correspond to BSD, SunOS, OS/2, AIX, HP-UX, and IRIX. Therefore, categorical attributes are highly skewed and their domains have only a few valid attribute values.

We further analyze the rate of change of attribute values, as it affects the resource advertising cost (Eq. (2)). There is a wide variation in how frequently the attribute values change. The number of changes in dynamic attributes over a 24-hour period is observed for two weeks. Figure 4 shows the CDF of number of significant changes in several selected attribute values. A fixed threshold is applied to ignore minor variations. MemFree of PlanetLab and GCO nodes changed frequently. For example, 54\% of the PlanetLab nodes changed MemFree by at least $100 \mathrm{MB}$ in every sample taken at 
Table IV. Distribution of Number of Significant Changes in Attribute Values within 24 Hours

\begin{tabular}{|l|l|l|}
\hline No of Changes in Attribute Values & PlanetLab* & \multicolumn{1}{c|}{ GCO } \\
\hline CPUFree & No fit & $G P D(0,34.4,-0.45)$ \\
\hline MemFree & No fit & $T L S(124,23,2.7)$ \\
\hline DiskFree & No fit & $T L S(14.2,6.86,3.84)$ \\
\hline 1MinLoad & No fit & $G E V(11.2,7.99,0.06)$ \\
\hline 15MinLoad & No fit & $G E V(2.57,2.93,0.31)$ \\
\hline TxRate & $N B(0.08,0.19)$ & $T L S(79.7,22.9,4.18)$ \\
\hline RxRate & $N B(0.09,0.19)$ & $T L S(142.9,51.6,13.7)$ \\
\hline
\end{tabular}

* GEV $(\mu, \sigma, k), G P D(\mu, \sigma, k), N B(r, p), T L S(\mu, \sigma, v)$

5-minute intervals, and 50\% of the GCO nodes changed MemFree at least 123 times within 24 hours (out of 288 samples taken at 5-minute intervals). While the number of changes in DiskFree was insignificant in PlanetLab and CSU datasets, it moderately changed in the GCO dataset. This may be due to the data incentive, high-energy physics applications that frequently run on these nodes [Germain-Renaud 2011b]. A relatively high rate of change was observed for 1 MinLoad in PlanetLab and CSU datasets and TxRate and RxRate in the GCO dataset. These rates indicate that the number of processes running on PlanetLab nodes shows a rapid variation with time, which could be either due to the variability in applications' resource usage or the execution of small jobs. The number of changes in 1MinLoad of CSU nodes was moderate; however, 15MinLoad changed infrequently, indicating most processes are short lived. Surprisingly, TxRate and RxRate do not change rapidly in PlanetLab; however, average bandwidth consumption of nodes remained relatively high. CPUFree moderately changed in the GCO dataset, as nodes were mostly busy. The number of processes running on nodes was stable with time, hence CPULoad changed infrequently. It was observed that the rate of change of some of the attributes could be approximated by probability distributions such as GPD, GEV, negative binomial (NB), and T locationscale (TLS) as listed in Table IV. In summary, rate of change $\lambda_{r}^{i}$ depends on the attribute and system.

Next, we analyze the linear and ranked correlation among attributes. Table V(a) lists the linear (Pearson's) correlation among PlanetLab nodes. For the dynamic attributes, we first calculated the correlation among all nodes in a given sample (taken at a given time instance) and then calculated their average across all the samples (taken at every 5 minutes over two weeks). Correlation values of most of the attribute pairs in different samples had a standard deviation of $\leq 0.1$, confirming that correlation does not significantly change with time. It can be seen that (15MinLoad, 1MinLoad), (DiskFree, NumCores), (DiskFree, MemSize), and (RxRate, TxRate) are positively correlated. 1MinLoad and 15MinLoad are correlated because they are the one-min and 15-min averages of CPULoad. Nodes with a large disk space also tend to have a large number of CPU cores and memory (correlation between NumCores and MemSize is 0.43). Time series of TxRate and RxRate are highly correlated as PlanetLab nodes tend to simultaneously transmit and receive data. Spearman's ranked correlation $\rho$ among attributes is listed in Table V(b). Spearman's $\rho$ measures how well the correlation between two attributes can be described using a monotonic function. The correlation between (CPUFree, NumCores), (MemFree, NumCores), and (MemFree, MemSize) have increased. This indicates that when a node has a large NumCores, CPUFree tends to increase, as it is the average of all the CPUs and some of them may be idle. It is also apparent that, when nodes have more CPU cores, their memory capacity also tends to increase. (1MinLoad, CPUFree) and (15MinLoad, CPUFree) are negatively correlated as CPUFree reduces when the number of processes using the CPU increases. 
Table V. Correlation among Attributes of PlanetLab Nodes (Values with Relatively High Correlation are Highlighted)

(a) Pearson's Correlation Coefficient

\begin{tabular}{|l|r|r|r|r|r|r|r|r|r|}
\hline Index* & \multicolumn{1}{c|}{1} & \multicolumn{1}{c|}{2} & \multicolumn{1}{c|}{3} & 4 & 5 & 6 & 7 & 8 & 9 \\
\hline 2 & -0.10 & & & & & & & & \\
\hline 3 & -0.01 & 0.46 & & & & & & & \\
\hline 4 & -0.03 & -0.03 & -0.16 & & & & & & \\
\hline 5 & -0.03 & -0.02 & -0.15 & 0.98 & & & & & \\
\hline 6 & 0.02 & 0.43 & 0.30 & -0.03 & -0.03 & & & & \\
\hline 7 & 0.06 & 0.25 & 0.35 & -0.12 & 0.10 & 0.36 & & & \\
\hline 8 & -0.11 & 0.56 & 0.41 & -0.09 & -0.09 & 0.58 & 0.30 & & \\
\hline 9 & 0.06 & -0.06 & -0.12 & -0.04 & -0.04 & 0.00 & 0.01 & -0.03 & \\
\hline 10 & 0.05 & -0.05 & -0.11 & -0.03 & -0.03 & 0.00 & 0.01 & -0.03 & 0.87 \\
\hline
\end{tabular}

(b) Spearman's Ranked Correlation Coefficient

\begin{tabular}{|l|r|r|r|r|r|r|r|r|r|}
\hline Index $^{*}$ & \multicolumn{1}{c|}{1} & \multicolumn{1}{c|}{2} & 3 & 4 & 5 & 6 & 7 & 8 & 9 \\
\hline 2 & 0.06 & & & & & & & & \\
\hline 3 & -0.09 & 0.68 & & & & & & & \\
\hline 4 & 0.08 & -0.20 & -0.62 & & & & & & \\
\hline 5 & 0.08 & -0.18 & -0.62 & 0.94 & & & & & \\
\hline 6 & -0.01 & 0.45 & 0.35 & -0.34 & -0.35 & & & & \\
\hline 7 & 0.20 & 0.61 & 0.51 & -0.03 & -0.03 & 0.69 & & & \\
\hline 8 & -0.21 & 0.66 & 0.56 & -0.42 & -0.42 & 0.44 & 0.60 & & \\
\hline 9 & 0.03 & 0.00 & -0.21 & 0.10 & 0.10 & 0.10 & 0.28 & -0.09 & \\
\hline 10 & 0.02 & 0.02 & -0.21 & 0.11 & 0.11 & 0.11 & 0.29 & -0.07 & 0.95 \\
\hline
\end{tabular}

* 1-CPUSpeed, 2 -NumCores, 3-CPUFree, 4-1MmLoad, 5-15MmLoad, 6-MemSize, 7 -MemFree, 8 -DiskFree, 9 - TxRate, 10 - RxRate

Table VI. Pearson's Correlation Coefficient among Attributes of GCO Nodes

\begin{tabular}{|l|c|c|r|r|r|r|r|r|c|}
\hline Index* & 1 & 2 & \multicolumn{1}{c|}{3} & \multicolumn{1}{c|}{4} & \multicolumn{1}{c|}{5} & 6 & 7 & 8 & 9 \\
\hline 2 & 1.00 & & & & & & & & \\
\hline 3 & 0.26 & 0.26 & & & & & & & \\
\hline 4 & 0.40 & 0.40 & -0.68 & & & & & & \\
\hline 5 & 0.42 & 0.42 & -0.67 & 0.96 & & & & & \\
\hline 6 & 1.00 & 1.00 & 0.26 & 0.40 & 0.42 & & & & \\
\hline 7 & 0.42 & 0.42 & 0.61 & -0.31 & -0.30 & 0.42 & & & \\
\hline 8 & 0.96 & 0.96 & 0.29 & 0.34 & 0.35 & 0.95 & 0.48 & & \\
\hline 9 & 0.03 & 0.03 & 0.01 & 0.04 & 0.04 & 0.03 & -0.01 & 0.03 & \\
\hline 10 & 0.12 & 0.12 & 0.03 & 0.08 & 0.07 & 0.12 & -0.06 & 0.10 & 0.25 \\
\hline
\end{tabular}

* 1 - CPUSpeed, 2 - NumCores, 3 - CPUFree, 4 - 1MmLoad, 5 - 15MmLoad, 6 -MemSize, 7 -MemFree, 8 -DiskFree, 9-TxRate, 10 - RxRate

Increased Spearman's $\rho$ values show that complex correlation patterns exist among attributes that are not necessarily linear. Similar behavior is also observed for SETI, GCO, and CSU nodes. Linear correlation of GCO nodes is listed in Table VI, where many attributes were correlated due to homogeneous nodes and heavy utilization of nodes (when a node is heavily utilized, CPUFree and MemFree reduce while CPULoad increases). Analysis of Spearman's $\rho$ also indicates TxRate and RxRate are positively correlated with a coefficient of 0.82 . 
Table VII. Composition of PlanetLab Queries

\begin{tabular}{|l|r|r|r|}
\hline & Attributes $\left(\boldsymbol{a}_{q}\right)$ & Resources $\left(\boldsymbol{m}_{q}\right)$ & Groups \\
\hline Average & 2.13 & 63.66 & 1.09 \\
\hline Minimum & 0 & 1 & 1 \\
\hline Maximum & 10 & All & 3 \\
\hline Median & 2 & 65 & 1 \\
\hline Mode & 1 & All & 1 \\
\hline Standard deviation & 1.62 & 380 & 0.3 \\
\hline
\end{tabular}

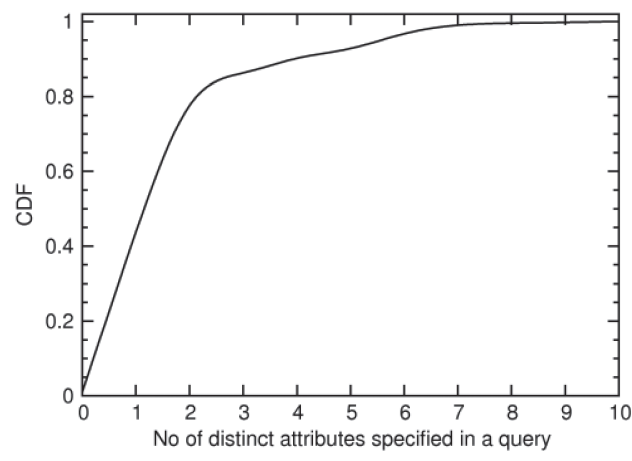

Fig. 5. Distribution of number of distinct attributes specified in a query.

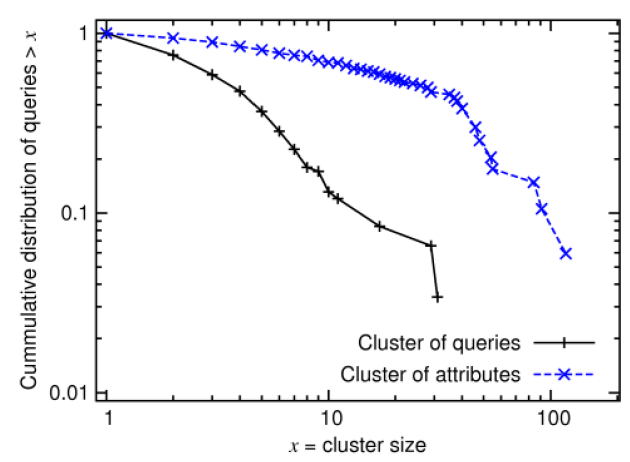

Fig. 7. Popularity distribution of queries and attributes specified in queries.

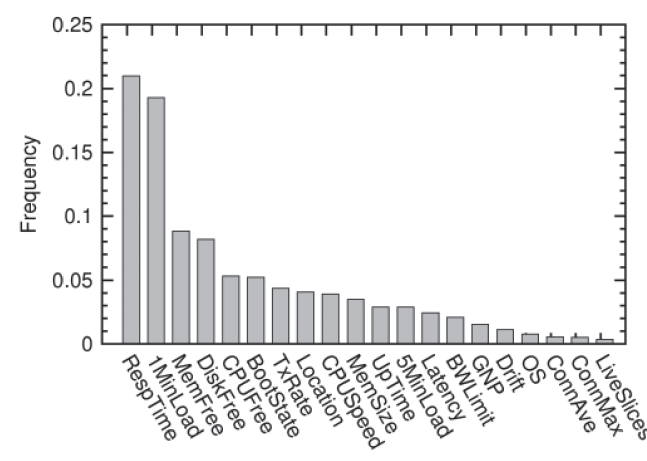

Fig. 6. Popularity of attributes specified in queries. Only the first 20 shown.

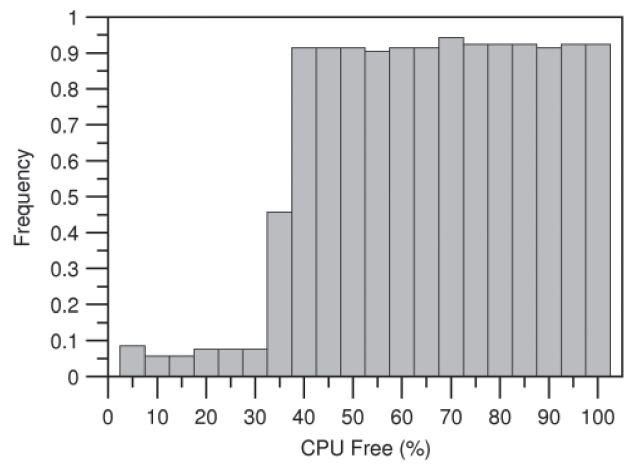

Fig. 8. Range of CPUFree values specified in queries.

\subsection{Query Characteristics}

Table VII summarizes the number of attributes $\left(a_{q}\right)$, resources $\left(m_{q}\right)$, and groups of resources specified in PlanetLab queries. Figure 5 shows the distribution of the number of attributes specified in a query. Specifically $2 \%$ of the queries requested a certain number of resources without specifying any attribute, while $78 \%$ of queries specified at most two attributes. Only $0.4 \%$ of queries specified 10 attributes. Thus, queries tend to specify a lower number of attribute values (i.e., $a_{q} \ll A$ ). There is also a tendency to request a large number of resources (i.e., large $m_{q}$ ), where $30 \%$ of queries requested ten resources while $52 \%$ requested 50 or more resources and $19 \%$ of queries requested all the resources in the system. Figure 6 shows the popularity of attributes specified in the queries. Dynamic attributes are the most popular. Specifically, 26 attributes (out of 49) were never queried. Many queries requested both CPUFree and CPUSpeed 
Table VIII. Summary of Resource and Query Characteristics

\begin{tabular}{|c|c|c|c|c|}
\hline Characteristics & PlanetLab & SETI@Home & GCO & CSU \\
\hline $\begin{array}{l}\text { Application } \\
\text { behavior }\end{array}$ & $\begin{array}{l}\text { Many small jobs, } \\
\text { variable CPU and } \\
\text { memory usage, } \\
\text { high bandwidth } \\
\text { utilization }\end{array}$ & $\begin{array}{l}\text { Long running } \\
\text { jobs }\end{array}$ & $\begin{array}{l}\text { Long running } \\
\text { jobs, high CPU, } \\
\text { memory, and } \\
\text { bandwidth } \\
\text { utilization }\end{array}$ & $\begin{array}{l}\text { Short lived jobs, } \\
\text { low CPU and } \\
\text { memory } \\
\text { utilization }\end{array}$ \\
\hline $\begin{array}{l}\text { Resource } \\
\text { attributes }\end{array}$ & $\begin{array}{l}\text { Correlated, some } \\
\text { identical resources }\end{array}$ & $\begin{array}{l}\text { Correlated, } \\
\text { heterogeneous }\end{array}$ & $\begin{array}{l}\text { Correlated, many } \\
\text { identical resources }\end{array}$ & $\begin{array}{l}\text { Correlated, many } \\
\text { identical resources }\end{array}$ \\
\hline$a_{r}$ & $\begin{array}{l}\text { Large, many } \\
\text { dynamic attributes }\end{array}$ & $\begin{array}{l}\text { Moderate, } \\
\text { no dynamic } \\
\text { attributes }\end{array}$ & $\begin{array}{l}\text { Large, many } \\
\text { dynamic attributes }\end{array}$ & $\begin{array}{l}\text { Moderate, } \\
\text { many dynamic } \\
\text { attributes }\end{array}$ \\
\hline $\mathbf{D}_{\mathbf{i}}$ & $\begin{array}{l}\text { Non-uniform, several } \\
\text { highly skewed } \\
\text { attributes, category- } \\
\text { cal attributes } \\
\text { with few values }\end{array}$ & -Do- & -Do- & -Do- \\
\hline$\lambda_{r}^{i}$ & $\begin{array}{l}\text { Very high for } \\
\text { several attributes }\end{array}$ & $\begin{array}{l}\text { Moderate due } \\
\text { to churn }\end{array}$ & $\begin{array}{l}\text { Very high for } \\
\text { several attributes }\end{array}$ & $\begin{array}{l}\text { Moderate for } \\
\text { several attributes }\end{array}$ \\
\hline$a_{q}$ & $\begin{array}{l}\text { Small, dynamic } \\
\text { attributes are } \\
\text { more popular }\end{array}$ & N/A & $\begin{array}{l}\text { Small, mostly } \\
\text { static attributes }\end{array}$ & $\begin{array}{l}\text { Small, mostly } \\
\text { dynamic attributes }\end{array}$ \\
\hline$\left[l_{i}, u_{i}\right]$ & Large, skewed & N/A & N/A & N/A \\
\hline$m_{q}$ & Large & N/A & Large & N/A \\
\hline
\end{tabular}

(or MemFree and MemSize). This may be because the percentage values (PlanetLab represents both CPUFree and MemFree as percentages) are inadequate to represent the actual availability of resources. We observed the query popularity by clustering identical queries together. Similarly, we clustered attributes with the same range of values. We found $24 \%$ of the queries and $13 \%$ of the attributes specified in queries appeared only once. The number of identical queries in the largest query cluster was 31 and the largest attribute cluster had 117 queries. Figure 7 shows the distribution of the cluster size. Though the popularity distribution is skewed, it does not satisfy a Pareto (or Zipf's-like) distribution as some of the queries are equally popular (curves do not approximate straight lines). It was further observed that the ranges of attribute values $\left[l_{i}, u_{i}\right]$ specified in queries are somewhat large. As seen in Figure $8,89 \%$ of the queries that specified CPUFree requested values of $40-100 \%$. Similarly, $86 \%$ of queries requested DiskFree values of 5-1000GB. Therefore, most queries are less specific as they specify a lower $a_{q}$ and large $\left[l_{i}, u_{i}\right]$. This could be due to several reasons: (1) the experimental applications (Table II) that run on PlanetLab may not require fine-grained resource selection, (2) users might have had an inability to identify detailed resource requirements, or (3) there may have been a principle of least effort [Brophy and Bawden 2005] where users are willing to specify only the simplest queries that satisfy their resource requirements.

\subsection{Summary of Resource and Query Characteristics}

Observed resource and query characteristics are summarized in Table VIII. Analysis of four datasets confirms that the different attribute values are correlated, marginal distributions of attributes are often quite different from one another, and in most cases are highly skewed and too complex to be represented using well-known probability distributions. Queries are skewed and less specific, as they specify small $a_{q}$, large $\left[l_{i}, u_{i}\right]$, and large $m_{q}$. These trends are likely to remain valid even in collaborative applications where users may not be informed enough to issue very specific queries, or due to the principle of least effort. Iosup and Epema [2010] also observed that, while most of the 
jobs in grids use a single CPU, a few tend to use a large number of CPUs (128 CPUs or more), and hence $m_{q}$ tends to be large even in grids. These findings invalidate the commonly used assumptions such as i.i.d. attributes, uniform/Zipf's distribution of all the attributes [Bharambe et al. 2004; Cai et al. 2004; Shen and Xu 2012], and queries specifying a large number of attributes and small ranges of attribute values [Cai et al. 2004; Shen and Xu 2012]. The correlation between attribute pairs indicates that resources are not uniformly distributed throughout the multi-attribute space (domain of all $\left.\mathbf{D}_{\mathbf{i}} \mathbf{s}\right)$. Domain $\mathbf{D}_{\mathbf{i}}$ 's of categorical attributes such as CPUArchi and $O S$ are small, as they have only a few distinct attribute values. Various hardware constraints favor having NumCores and MemSizes that are powers of two. Therefore, these attributes occupy only a small fraction of their $\mathbf{D}_{\mathbf{i}}$ s. Moreover, large numbers of identical resources in PlanetLab, grid, cloud, and enterprise computing environments appear as sets of clusters within the multi-attribute space.

Both $a_{r}$ and $A$ are large as the resources are described using tens of attributes (see Table II). Dynamic attributes change at different rates and some frequently (high $\lambda_{r}^{i}$ ). A user can decide when to run a BOINC client. Hence, overall availability of SETI nodes is $68 \%$ and nodes were reachable through the network $67 \%$ of the time [Anderson and Fedak 2006]. Alternatively, most nodes in PlanetLab, GCO, and CSU datasets were available throughout the two-week period. Thus, the availability of nodes in volunteer computing environments is significantly lower compared to more stable environments like PlanetLab, grids, and clouds. Consequently, $\lambda_{r}^{i}$ increases further due to frequent arrival and departure of nodes. It is also observed that grids tend to experience sudden arrivals of sets of jobs (a.k.a. a bag of tasks) [Iosup and Epema 2010]. Such arrivals suddenly change the dynamic attributes of many resources.

As the real-world resources and queries diverge substantially from the conventional assumptions, it is imperative to understand how existing P2P-based RD solutions will perform in real systems. Based on our findings from this section, next we evaluate a representative subset of design choices for P2P-based RD. In Section 5, we evaluate the asymptotic behavior of RD solutions based on real-world parameters. In the subsequent section, we also empirically evaluate RD solutions using resource and query traces from PlanetLab.

\section{DESIGN CHOICES IN P2P-BASED RESOURCE DISCOVERY}

To ease the RD, resources are typically advertised and indexed at specific or random locations within the RD system. Resources are then located using various querying mechanisms. Resource advertising and querying options of centralized, unstructured $\mathrm{P} 2 \mathrm{P}$, and structured $\mathrm{P} 2 \mathrm{P}$ solutions are analyzed next using the characteristics learned in the previous section. Eq. (6) is also extended to reflect the specific behavior of each solution.

\subsection{Centralized Designs}

Resources can be advertised and indexed at a well-known central location. Centralized indexes are utilized in PlanetLab, GENI, and grid and cloud computing. Latency and cost of resource advertising and querying are minimum, as nodes can directly communicate with the central node. Hence, the cost per message is $O(1)$. Resolving queries with large $\left[l_{i}, u_{i}\right]$ and $m_{q}$ does not introduce additional routing overhead. Thus, the $\mathrm{RD}$ cost depends on the initial advertisement cost, recurrent cost of advertising static/dynamic attributes, and query cost. Then (6) can be modified as

$$
C_{\text {Total }}=\sum_{r \in \mathbf{R}}\left(1+\sum_{j \in \mathbf{a}_{\mathbf{r}}} \lambda_{r}^{j} t\right)+Q(t),
$$


where $c_{r}^{i}=1$, as resources are advertised only to the central location. If the query arrival rate is constant, the total cost of $\mathrm{RD}$ is bounded by $O\left(R A \lambda_{r}^{i}\right)$. In the worst case, all the resources may have all the attributes. Therefore, the cost of indexing resources at a central node is $O(R A)$. This approach is not scalable (in terms of both the number of messages and index size) as $A, R$, and $\lambda_{r}^{i}$ are typically large in production systems. It also leads to a single point of failure.

Hierarchical indexes are proposed to overcome these limitations where separate indexing nodes are assigned to different geographic regions, sites, or organizations [Elliott 2009]. Then a higher-level node(s) is assigned to keep track of aggregated resources from these nodes. However, aggregation along the hierarchy reduces the resolution at which resources are advertised. Therefore, such hierarchies are not desirable for indexing highly dynamic attributes such as MemFree and bandwidth. This approach could also lead to conflicts while querying and binding resources. Partial failures in subregions of the system are also problematic.

\subsection{Unstructured P2P-Based Designs}

Unstructured P2P systems [Lua et al. 2004] are attractive as they distribute the resource information across many nodes in the system while providing resilience and load balancing. They are utilized in file sharing, mobile social networks, and adhoc networks. These systems use either flooding or random walks [Tan et al. 2008] to disseminate information about resources and/or resolve queries. Though resources are guaranteed to be found with flooding, it is extremely costly. Random-walk-based solutions use agents for advertising and/or querying. Agent lifetime is controlled using a time-to-live $\left(h_{T T L}\right)$ value that tries to balance the cost of advertising/querying and query success rate. As queries are forwarded to individual nodes, their availability is known. Hence, no additional overhead is introduced. Assuming a new agent is generated for each change in attribute value or query, the RD cost is

$$
C_{T O T A L}=\sum_{r \in \mathbf{R}}\left(\sum_{i \in \mathbf{a}_{\mathbf{r}}} h_{T T L}+\sum_{j \in \mathbf{a}_{\mathbf{r}}} \lambda_{r}^{j} t h_{T T L}\right)+\sum_{q \in \mathbf{Q}(\mathbf{t})} h_{Q u e r y}^{q}
$$

where $h_{\text {Query }}^{q}$ is typically $O\left(h_{T T L}\right)$. Therefore, the total cost is bounded by $O\left(R A \lambda_{r}^{i} h_{T T L}\right)$. However, random walks are not guaranteed to find resources, and this will become even harder if one reduces $h_{T T L}$ with the intention of reducing the cost of RD. It has been shown that the hitting time (i.e., expected number of hops to reach any node staring at any given node) of a random walk on an arbitrary finite graph is $O\left(n^{3}\right)$ [Ikeda et al. 2009]. Hence, $h_{T T L}$ has to scale with $n^{3}$ to increase the success rate of queries. Therefore, unstructured $\mathrm{P} 2 \mathrm{P}$ systems are suitable only for moderate-scale, best-effort, and highly dynamic environments that can tolerate large delays. Resource indexing cost per node is $O(R A)$. Though advertising resources to other nodes speeds up the query resolution, the state of the selected resources may be stale.

A more scalable version of this approach uses a two-layer overlay where resource rich nodes, namely superpeers, form a separate overlay while acting as proxies for the rest of the nodes. Similar to hierarchical solutions, superpeers manage multiple resources. Resources can communicate with their superpeers using one overlap hop. When local resources are insufficient to resolve a query, superpeers query other superpeers using flooding or random walks. Therefore $h_{\text {Query }}^{q}=O\left(n_{\text {Superpeer }}\right)$ or 


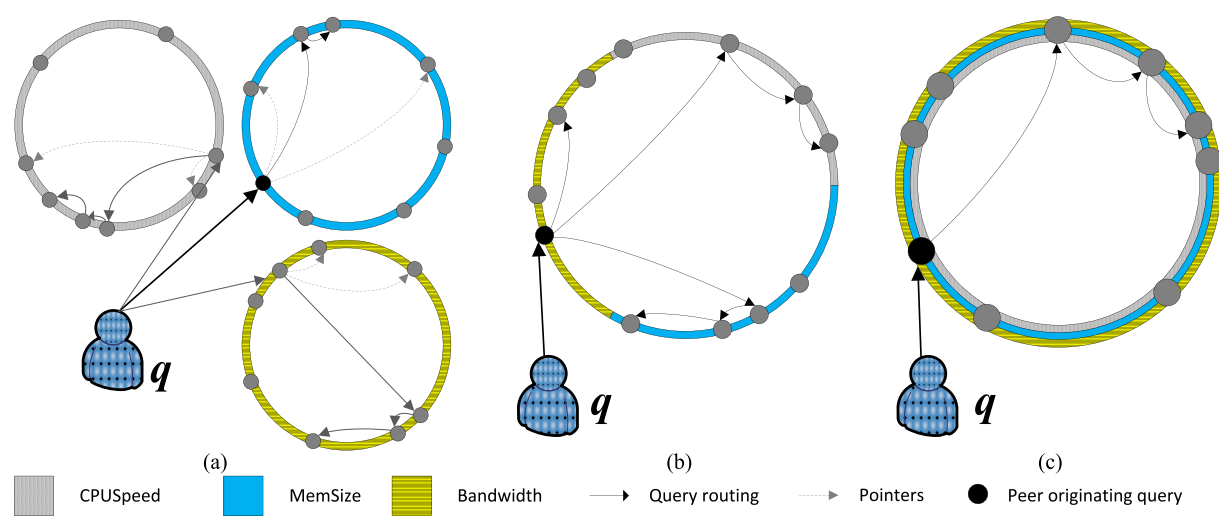

Fig. 9. Ring-based address spaces: (a) Separate address space for each attribute and single-attributedominated query resolution; (b) address space partitioned across multiple attributes and query resolution with multiple subqueries; (c) overlapped address space and single-attribute-dominated query resolution.

$h_{Q u e r y}^{q}=\mathrm{O}\left(h_{T T L}\right)$, where $n_{\text {Superpeer }}$ is the number of superpeers in the system. The cost of $\mathrm{RD}$ is

$$
C_{\text {TOTAL }}=\sum_{r \in \mathbf{R}}\left(1+\sum_{i \in \mathbf{a}_{\mathbf{r}}} \lambda_{r}^{i} t\right)+\sum_{q \in \mathbf{Q}(\mathbf{t})} h_{\text {Query }}^{q} .
$$

As $n_{\text {Superpeer }} \ll n, h_{T T L}$ can be set to a relatively lower value than in unstructured P2P systems. However, superpeers are also not guaranteed to find resources with random walks. Indexing load on a superpeer is proportional to $A$ and the number of resources to which it connects. If resources are uniformly assigned to superpeers, the index size is $O\left(R A / n_{\text {Superpeer }}\right)$.

\subsection{Structured P2P-Based Designs}

Structured P2P systems are appropriate for large-scale implementations due to their high scalability and their ability to provide some guarantees on performance [Lua et al. 2004]. These systems use a DHT to index resources. Each DHT node or resource has a unique identifier called a key. Each resource's contact information is indexed (i.e., stored) at a node having a closeby key in the key space. Resources are advertised and queried using messages that are forwarded to appropriate nodes using a deterministic overlay network. Chord, Kademlia, CAN, and Pastry are some of the well-known solutions that are used to build such an overlay [Lua et al. 2004]. These solutions typically keep pointers to nodes that are spaced at exponentially increasing gaps in the key space, enabling messages to be routed with a bounded path length of $O(\log n)$. DHTs are designed to index resources characterized by a single attribute. As such, they are not efficient for simultaneous selection of multiple and multi-attribute resources. Four solutions representative of methods that extend DHTs to support multi-attribute RD are discussed next.

Multiple Address Spaces. As seen in Figure 9, ring-based DHTs can be extended to support multiple attributes. One of the simplest solutions is to maintain a separate DHT for each attribute in $A$ [Bharambe et al. 2004]. Figure 9(a) illustrates a design based on three DHTs with a circular address space, also referred to as a ring. Each resource $r$ advertises either each attribute value to the corresponding ring or all the attribute values to all the rings responsible for $\mathbf{a}_{\mathbf{r}}$. In the former case, a multi-attribute query $q$ is first split into a set of subqueries where each subquery searches for one of the 
attributes in $\mathbf{a}_{\mathbf{q}}$. Subqueries are then simultaneously forwarded to appropriate rings. Query results have to be then combined at the application using a join operation like that in databases. A range query is resolved by forwarding each subquery to a series of nodes responsible for indexing attribute values in the range $\left[l_{i}, u_{i}\right]$. Thus, the RD cost is

$C_{\text {TOTAL }}=\sum_{r \in \mathbf{R}}\left(\sum_{i \in \mathbf{a}_{\mathbf{r}}} h_{\text {Advertise }}^{i}+\sum_{j \in \mathbf{a}_{\mathbf{r}}} h_{\text {Advertise }}^{j} \lambda_{r}^{j} t\right)+\sum_{q \in \mathbf{Q}(\mathbf{t})}\left\{\sum_{i \in \mathbf{a}_{\mathbf{q}}}\left(h_{\text {Query }}^{i}+\left\lceil\frac{\left(u_{i}-l_{i}\right)}{D_{i}} n\right\rceil-1\right)\right\}$.

Each advertising message can be sent in $O(\log n)$ hops, and thus the advertising cost is $O\left(R A \lambda_{r}^{i} \log n\right)$. Worst-case query cost is $O(n)$ as $h_{\text {Query }}^{i}=O(\log n)$. Even the average cost is large, as queries tend to specify large $\left[l_{i}, u_{i}\right]$. The indexing cost of all the rings is $O(R A)$ or $O(R)$ per ring. In the latter case, $q$ can be resolved using one of the rings, as each ring is aware of all the attribute values of a resource. This also enables queries to be terminated as soon as the desired number of resources is found. Query resolution cost can be further reduced by forwarding $q$ to the ring corresponding to the most selective attribute (i.e., attribute with the lowest $\left.\left(u_{i}-l_{i}\right) / D_{i}\right)$. For example, as seen in Figure 9(a), the query travels 3 hops in CPUSpeed and bandwidth rings, while only 2 hops in the MemSize ring. Thus, $q$ is issued only to the MemSize ring. This approach is called single-attribute-dominated querying (SADQ) and used in Albrecht et al. [2008], Bharambe et al. [2004], and Cai et al. [2004]. Total RD cost in this case is

$C_{\text {TOTAL }}=\sum_{r \in \mathbf{R}}\left(\sum_{i \in \mathbf{a}_{\mathbf{r}}} h_{\text {Advertise }}^{i}+\sum_{j \in \mathbf{a}_{\mathbf{r}}} h_{\text {Advertise }}^{j} \lambda_{r}^{j} a_{r} t\right)+\sum_{q \in \mathbf{Q}(\mathbf{t})}\left(h_{\text {Query }}^{k}+\left\lceil\frac{\left(u_{k}-l_{k}\right)}{D_{k}} n\right\rceil-1\right)$,

where $k \in \mathbf{a}_{\mathbf{q}}$ is the most selective attribute. In the worst case, each change in attribute values has to be advertised to all the rings, resulting in an advertising cost of $O\left(R A^{2} \lambda_{r}^{i} \log n\right)$. Worst-case query cost is still $O(n)$. Real-world queries specify only a few attributes, and hence not many options are available while choosing the most selective attribute. Therefore, even the average query cost is relatively high. Indexing cost of all the rings is $O\left(R A^{2}\right)$, or $O(R A)$ per ring. It has a lower query cost than using multiple subqueries. However, it has a higher advertising cost due to the presence of multiple copies where $c_{r}^{i}=a_{r}$ (recall that $a_{r}$ tends to be large). Therefore, it is suitable only if the query rate is higher than $\lambda_{r}^{i}$. New rings can be added to support additional attributes. However, excessive routing entries associated with multiple rings (typically $O(A \log n)$ make it less scalable. Some rings experience higher load due to skewed distribution of resources and queries. This is particularly a problem in SADQ where highly selective and skewed attributes such as CPUArchi, NumCores, and OS may get a large number of queries overloading the nodes that index them. Moreover, domain $\mathbf{D}_{\mathbf{i}}$ of these attributes is small and hence may not be suitable to be placed on a separate ring/overlay.

Partitioned Address Space. Figure 9(b) shows a ring partitioned into several segments where each segment corresponds to a separate range of attribute values. Prefix bits of the overlay key are used to represent the attribute type and suffix bits represent the attribute value. A locality-preserving hash (LPH) function [Albrecht et al. 2008; Shen and Xu 2012] is used to assign the suffix bits, which preserves the locality of attribute values. Advertising and querying schemes are similar to the case of multiple address spaces. However, each partition has approximately $n / A$ nodes, 


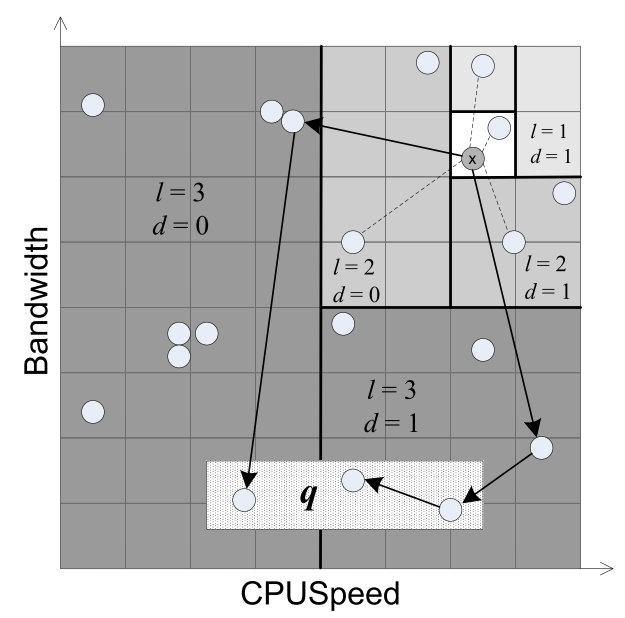

Fig. 10. 2D torus partitioned into a set of virtual cells with respect to a given node $x$. A query is routed to all nodes within the query hyperbox. Here $l$ is the level, and $d$ the dimension [Costa et al. 2009].

therefore worst-case query cost is $O(n / A)$. Moreover, these solutions maintain a much lower number of routing entries, typically $O(\log n)$. Some partitions experience higher load due to skewed distribution of resources and queries while most of the remaining partitions are rarely utilized.

Overlapped Address Space. Another alternative is to map all the attribute values to the same ring (see Figure 9(c)) using a separate LPH function for each attribute type [Cai et al. 2004]. The cost of RD and indexing is the same as that with multiple address spaces because the same advertising and querying mechanisms are applicable here. Some nodes experience higher loads due to skewness in resources and queries. However, the routing state is $O(\log n)$ as only a single ring is used.

$d$-Torus. Figure 10 illustrates an alternative design where each dimension of a $d$ torus represents an attribute (i.e., $d=A$ ). Resources are mapped to the torus according to their attribute values. A multi-attribute range query $q$ encloses a hyperbox on the torus. This approach is used in Costa et al. [2009], where dimensions of the torus reflect only the static attributes (i.e., $d=A^{s}$ ). To facilitate routing, the $d$-torus is partitioned into an increasing set of levels, each further partitioned along each dimension $d$, forming a set of cells. Each node keeps a set of pointers to all the other nodes in the same cell and to a node in each partition at level $l$ and dimension $d$. In contrast to prior architectures, resources are not explicitly advertised. Instead, a gossip scheme is used to identify a random node in each $(l, d)$ pair. These pointers are used to route $q$ to the desired query region on the torus using depth-first search (see Figure 10). Then neighboring nodes are used to traverse from one node to another, during which dynamic attributes in $q$ may be evaluated. The RD cost for $d$-torus is therefore

$$
C_{\text {TOTAL }}=\sum_{q \in \mathbf{Q}(\mathbf{t})} h_{\text {Query }}^{q}+m_{q}-1
$$

It can be shown that $h_{\text {Query }}^{q}$ is $O\left(\ln 2^{d} / \ln d\right)$. After reaching the first node that satisfies $q$, the remaining $m_{q}-1$ resources need to be found. Hence, the worst-case query cost is $\mathrm{O}\left(\ln 2^{d} / \ln d+m_{q}-1\right)$, ignoring the overlay maintenance cost. As the propagation of $q$ is based on depth-first search and the query visits individual nodes (one at a time), query resolution latency is much higher. This routing scheme is inefficient when 
Table IX. Summary of Resource Discovery Architectures

\begin{tabular}{|c|c|c|c|c|c|c|c|}
\hline \multirow{2}{*}{ Architecture } & \multirow{2}{*}{$\begin{array}{c}\text { Routing } \\
\text { Mechanism }\end{array}$} & \multicolumn{2}{|c|}{ Advertising } & \multicolumn{2}{|c|}{ Querying } & \multicolumn{2}{|c|}{ Indexing } \\
\hline & & Mechanism & Cost & Mechanism & Cost & Mechanism & Cost \\
\hline Centralized & Direct & $\begin{array}{l}\text { To central } \\
\text { node }\end{array}$ & $O(1)$ & $\begin{array}{l}\text { Query } \\
\text { central node }\end{array}$ & $O(1)$ & $\begin{array}{l}\text { At central } \\
\text { node }\end{array}$ & $O(R A)$ \\
\hline $\begin{array}{l}\text { Unstructured- } \\
\text { Random overlay }\end{array}$ & $\begin{array}{l}\text { Random } \\
\text { walk }\end{array}$ & Optional & $O(h T T L)$ & $\begin{array}{l}\text { Query } \\
\text { random } \\
\text { nodes }\end{array}$ & $O(h T T L)$ & $\begin{array}{l}\text { Index } \\
\text { locally }\end{array}$ & $\begin{array}{l}O(A) \text { or } \\
O(R A) \text { if } \\
\text { advertised }\end{array}$ \\
\hline $\begin{array}{l}\text { Superpeer - } \\
\text { Random } \\
\text { overlay among } \\
\text { superpeers }\end{array}$ & $\begin{array}{l}\text { Random } \\
\text { walk among } \\
\text { superpeers }\end{array}$ & $\begin{array}{l}\text { To } \\
\text { superpeer }\end{array}$ & $O(1)$ & $\begin{array}{l}\text { Query } \\
\text { superpeers }\end{array}$ & $O(h T T L)$ & $\begin{array}{l}\text { At } \\
\text { superpeer }\end{array}$ & $\begin{array}{l}O(R A / \\
\left.n_{\text {Superpeer }}\right)\end{array}$ \\
\hline $\begin{array}{l}\text { Multi-ring - } \\
\text { Separate } \\
\text { ring-like } \\
\text { overlay } \\
\text { for each } \\
\text { attribute } \\
\text { type }\end{array}$ & Chord & $\begin{array}{l}\text { To relevant } \\
\text { ring(s) based } \\
\text { on attributes }\end{array}$ & $\mathrm{O}(\log n)$ & $\begin{array}{l}\text { 1. Multiple } \\
\text { sub-queries } \\
\text { 2. Single } \\
\text { attribute } \\
\text { dominated } \\
\text { query }\end{array}$ & $O(n)$ & $\begin{array}{l}\text { 1. At } \\
\text { relevant } \\
\text { rings } \\
2 \text {. At all } \\
\text { rings }\end{array}$ & $\begin{array}{l}\text { 1. } O(R A) \\
\text { 2. } O\left(R A^{2}\right)\end{array}$ \\
\hline $\begin{array}{l}\text { Partitioned- } \\
\text { ring - Each } \\
\text { attribute type } \\
\text { is assigned } \\
\text { a different } \\
\text { segment of } \\
\text { overlay ring }\end{array}$ & $\begin{array}{l}\text { Chord or } \\
\text { Cycloid }\end{array}$ & $\begin{array}{l}\text { To relevant } \\
\text { partition }(\mathrm{s}) \\
\text { based on } \\
\text { attributes }\end{array}$ & $\mathrm{O}(\log n)$ & $\begin{array}{l}\text { 1. Multiple } \\
\text { sub-queries } \\
\text { 2. Single } \\
\text { attribute } \\
\text { dominated } \\
\text { query }\end{array}$ & $O(n \mid A)$ & $\begin{array}{l}\text { 1. At } \\
\text { relevant } \\
\text { partitions } \\
2 . \text { At all } \\
\text { partitions }\end{array}$ & $\begin{array}{l}\text { 1. } O(R A) \\
\text { 2. } O\left(R A^{2}\right)\end{array}$ \\
\hline $\begin{array}{l}\text { Overlapped- } \\
\text { ring - All } \\
\text { attribute types } \\
\text { are mapped to } \\
\text { same ring-like } \\
\text { overlay }\end{array}$ & Chord & $\begin{array}{l}\text { To relevant } \\
\text { nodes in the } \\
\text { ring }\end{array}$ & $\mathrm{O}(\log n)$ & $\begin{array}{l}\text { Single } \\
\text { attribute } \\
\text { dominated } \\
\text { query }\end{array}$ & $O(n)$ & $\begin{array}{l}\text { At relevant } \\
\text { nodes }\end{array}$ & $O\left(R A^{2}\right)$ \\
\hline $\begin{array}{l}d \text {-Torus - } \\
d \text {-torus } \\
\text { partitioned } \\
\text { into set of cells }\end{array}$ & $\begin{array}{l}\text { Depth-first } \\
\text { search }\end{array}$ & Not required & - & $\begin{array}{l}\text { Visit cells } \\
\text { that overlap } \\
\text { with query } \\
\text { hyper-box }\end{array}$ & $O\left(\frac{\ln 2^{d}}{\ln d}\right)$ & $\begin{array}{l}\text { Index } \\
\text { locally }\end{array}$ & $O(A)$ \\
\hline
\end{tabular}

queries are less specific, which significantly increases the volume of the hyperbox to search. Moreover, it cannot route queries with only the dynamic attributes, which accounts for a large fraction of queries in real-world systems (Figure 6). A comparative summary of all the RD solutions is provided in Table IX. In the next section, we verify the asymptotic behavior by empirically evaluating these RD solutions using the resource and query traces from PlanetLab.

\section{PERFORMANCE ANALYSIS}

Next, we quantitatively evaluate the fundamental design choices for P2P-based RD. We simulated seven representative architectures for RD (listed in Table IX) against the same set of resources and queries derived from PlanetLab (see Bandara [2012] for source code). The use of realistic data preserves the complex distribution of attributes, dynamic and correlated changes in attribute values, and users' interest in resources. To simplify the performance analysis and eliminate any bias due to node failure, we replayed a trace with only those PlanetLab nodes that were continuously available for three days starting 2010/11/08. There were 527 such nodes. As our query dataset was small, a large number of synthetic queries were generated using the empirical distributions of $a_{q}$, popularity of attributes, $\left[l_{i}, u_{i}\right]$, and $m_{q}$ [Bandara 2012]. To capture the correlation among attributes and attribute value ranges, conditional probabilities of attribute occurrences were also taken into account. Queries were issued only after the network was stabilized.

Both the unstructured and superpeer-based networks were generated using the B-A scale-free network generator [George 2006] with a minimum node degree of two. Advertising dynamic attributes is not that useful in unstructured $\mathrm{P} 2 \mathrm{P}$ architectures and 


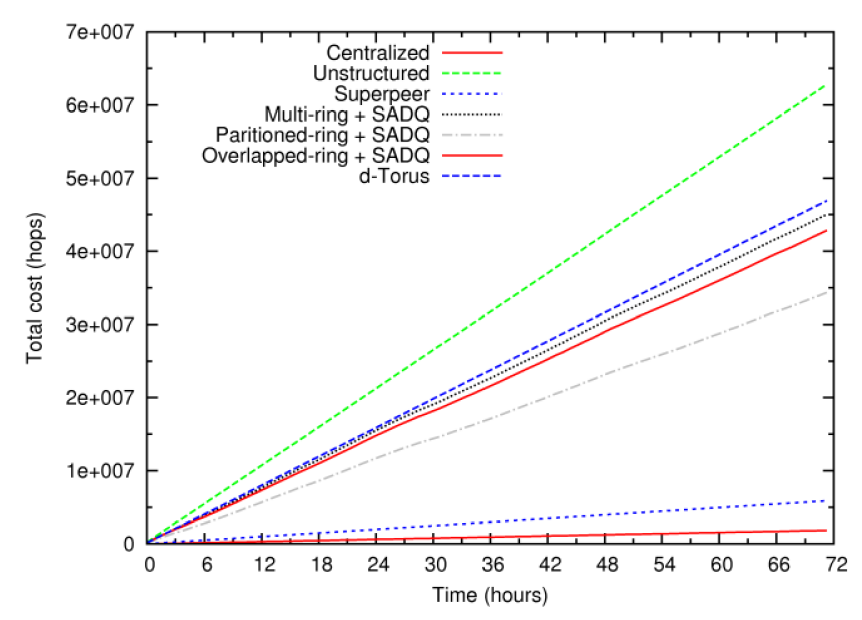

Fig. 11. Total cost of advertising and querying static attributes $(n=527)$.

among superpeers in superpeer architectures. As random walks are not guaranteed to follow the same path, a new advertising agent may not be able to overwrite the resource states advertised by previous agents. Hence, nodes may not reflect the current status of resources by the time they are queried. Therefore, only the query agents were used in these two solutions. The number of superpeers is set to 20 . The maximum number of hops to forward a random walk (i.e., $h_{T T L}$ ) was set to 100 and 10 hops in unstructured and superpeer architectures, respectively. These values were sufficient to achieve $\sim 70 \%$ query hit rate with different numbers of attributes. Multiring, partitioned ring, and overlapped ring architectures were based on the Chord overlay [Stoica et al. 2003]. According to Costa et al. [2009], the number of cell levels of the $d$-torus was set to three. Performance of ring-based structured P2P solutions was also evaluated under a different number of nodes ranging from 250 to 1000 . Resource attributes for 750- and 1000-node networks were generated using our correlation-persevering, multi-attribute resource generation tool [Bandara and Jayasumana 2011a]. The tool uses the statistical properties of 527 PlanetLab nodes to generate large synthetic datasets. The minimum update interval for resource attributes is $5 \mathrm{~min}$, which is the sampling interval of PlanetLab traces. Fixed thresholds given in Figure 4 were applied to ignore minor variations. Each node issued queries based on a Poisson distribution with a mean inter-arrival time of 2.5 min (i.e., two queries per sampling interval per node). Though we consider a modest number of nodes for the simulations, we analyze them in much more detail by considering many different attributes, their dynamic behavior over three days, and many queries. Networks with 100000 nodes, under a more constrained set of attributes, are analyzed in Bandara and Jayasumana [2012c] and Bandara [2012]. Results are based on eight sample runs with eight different random seeds.

Figure 11 shows the total cost of advertising and querying resources using only the 12 static attributes of PlanetLab nodes (see Bandara [2012] for details). This enables to validate the performance of different architectures against their prior performance studies (when such a study exists) which did not consider the cost of advertising dynamic attributes. As expected, a centralized architecture has the lowest overall cost while the unstructured P2P architecture has the highest. The superpeer-based architecture has the second-lowest cost. Less specific queries (large $\left[l_{i}, u_{i}\right]$ and small $a_{q}$ ) make it easier to find required resources by visiting a few superpeers that index 


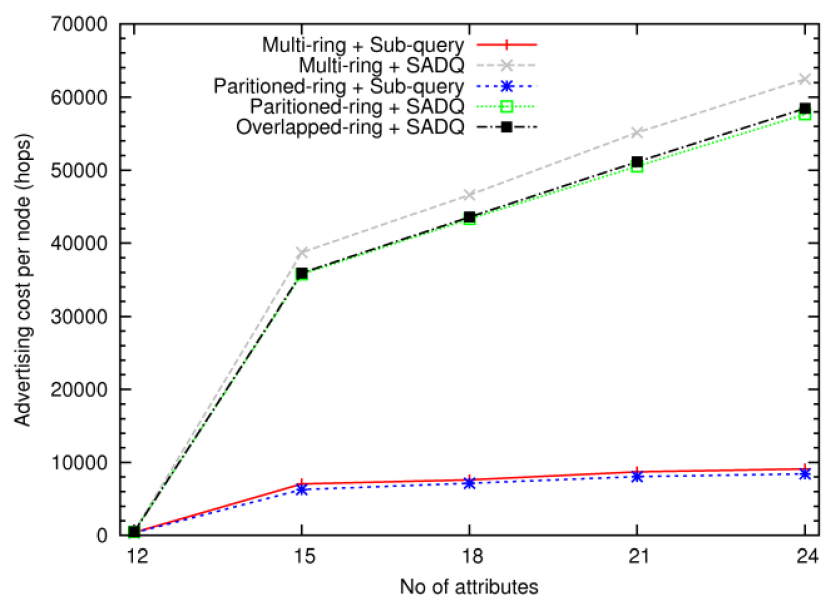

Fig. 12. Advertising cost of ring-based architectures $(n=527)$.

multiple resources. For example, though random walks are not granted to find all the resources, the superpeer architecture was able to resolve $96 \%$ of the queries. Therefore, most of the random walks were able to terminate within a few hops while reducing the overall cost. However, the unstructured P2P solution was able to resolve only $73 \%$ of the queries, as they visit one node at a time. The partitioned ring-based architecture has the third-lowest cost, as query cost is $O(n / A)$. Costs of multiring and overlapped ring-based architectures are higher compared to the partitioned ring, as their query cost is $O(n)$. And $d$-torus has the second-largest overall cost due to less-specific queries that dramatically increase the volume of the query hyperbox. It is not considered for rest of the discussion, as it cannot route those queries with only dynamic attributes, which are the most popular types of attributes defined in real-world, multi-attribute queries.

We analyze ring-based architectures in detail, as they are considered applicable in large-scale applications due to scalability and some guarantees on performance. Figure 12 shows the per-node advertising cost of ring-based architectures under a varying number of attributes. Advertising cost increases as the dynamic attributes are introduced (the first 12 attributes are static). Resources need to be re-advertised whenever their attribute values change significantly. It is typically assumed that DHT entries will expire after a predefined timeout. However, our analysis shows that it is nontrivial to determine an appropriate timeout given the diversity of attributes and their rate of change $\lambda_{r}^{i}$. Therefore, the old attribute values need to be explicitly removed from the DHT to maintain a consistent resource index. We considered the cost of removing these old indexes as part of the advertising cost. Both advertise and remove messages can be delivered within $O(\log n)$ as they are sent to specific nodes. SADQ requires all the attributes of a resource to be advertised to each ring/partition corresponding to each attribute $a \in \mathbf{a}_{\mathbf{r}}$. Therefore, resources need to be re-advertised to all the rings/partitions even when a single attribute is changed. Figure 12 confirms this behavior where the advertising cost of architectures that utilize SADQ is significantly higher and increases somewhat linearly with the number of attributes. We introduced dynamic attributes according to their popularity, where attribute 13 is the most popular, 14 is the second most popular, and so on. Attributes 13-15 that correspond to the response time of a node (RespTime), 1MinLoad, and MemFree are updated frequently. This is the reason for the significant increase in advertising cost between attributes 12 and 15 . The $\lambda_{r}^{i}$ of the rest of the dynamic attributes was relatively lower. 


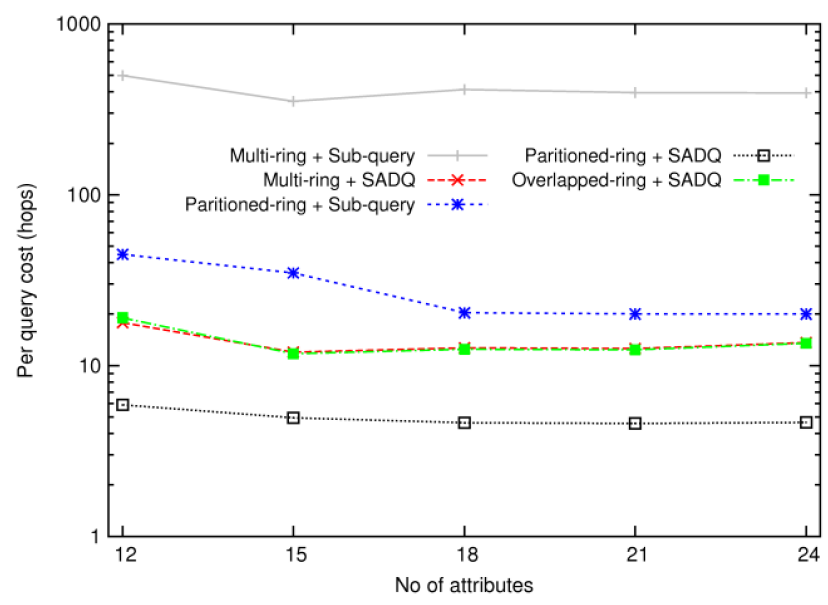

Fig. 13. Query cost of ring-based architectures $(n=527)$.

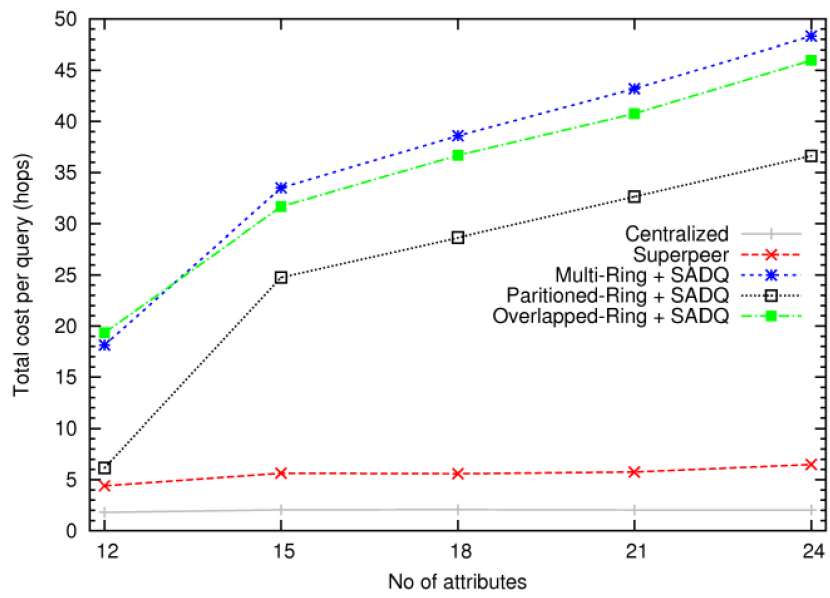

Fig. 14. Total cost (advertising + querying) vs. number of attributes $(n=527)$.

Figure 13 shows the cost per query. Architectures based on SADQ have much lower query cost, as they use only the most selective attribute and queries are terminated as soon as $m_{q}$ resources are found. However, overall cost (advertising and querying) of SADQ-based architectures will be acceptable only if queries are more frequent than advertisements. Alternatively, subqueries need to search in multiple rings/partitions and have to search the entire range of attribute values specified in each subquery, hence have higher cost. Queries that specified attributes 13 and 14 (RespTime and 1 MinLoad) tend to be relatively more specific (i.e., small $\left.\left(u_{i}-l_{i}\right) / D_{i}\right)$ therefore can be resolved by forwarding to a lesser number of nodes. Furthermore, these were the two most popular attributes, hence appeared in many queries and consequently reducing the overall query cost. Therefore, query cost drops when the number of attributes is 15. Query distribution and range of attribute values get balanced as the rest of the dynamic attributes are introduced. Consequently, query cost tends to stabilize. Though new attributes were introduced, PlanetLab queries specified only 1-10 attributes and one or two attributes were specified $78 \%$ of the time. This explains why query cost seems independent of the number of attributes in the system. 
Table X. Query Cost of Ring-Based Designs under Varying Number of Nodes $(A=24)$

\begin{tabular}{|l|c|c|c|c|c|c|c|c|c|}
\hline $\boldsymbol{n}$ & \multicolumn{3}{|c|}{ Multi-Ring + SADQ } & \multicolumn{3}{c|}{ Partitioned-Ring + SADQ } & \multicolumn{3}{c|}{ Overlapped-Ring + SADQ } \\
\hline & Min & Ave & Max & Min & Ave & Max & Min & Ave & Max \\
\hline 250 & 0 & 9.2 & 239.1 & 0 & 3.7 & 19.4 & 0 & 9.1 & 238.4 \\
\hline 527 & 0 & 13.7 & 509.0 & 0 & 4.6 & 27.6 & 0 & 13.5 & 506.0 \\
\hline 750 & 0 & 16.2 & 719.1 & 0 & 4.9 & 36.6 & 0 & 16.5 & 719.9 \\
\hline 1000 & 0 & 19.8 & 975.5 & 0 & 5.3 & 45.3 & 0 & 20.4 & 963.8 \\
\hline
\end{tabular}

Table XI. Query Cost, Query Load, and Index Size $(n=527, A=24)$

\begin{tabular}{|c|c|c|c|c|c|c|c|c|}
\hline \multirow{3}{*}{ Architecture } & \multirow{2}{*}{\multicolumn{2}{|c|}{ Total Cost per Query }} & \multicolumn{4}{|c|}{ Query Load } & \multicolumn{2}{|c|}{ Index Size } \\
\hline & & & \multicolumn{2}{|c|}{ Min } & \multicolumn{2}{|c|}{$\operatorname{Max}$} & \multirow{2}{*}{ Min } & \multirow{2}{*}{ Max } \\
\hline & SWORD & Uniform & SWORD & Uniform & SWORD & Uniform & & \\
\hline Centralized & 2.03 & 2.03 & 950,000 & 950,000 & 950,000 & 950,000 & 527 & 527 \\
\hline Unstructured & 69.5 & 94.8 & 4,859 & 1,272 & 268,497 & 37,824 & 1 & 1 \\
\hline Superpeer & 6.5 & 9.5 & 81,021 & 22,390 & 289,626 & 87,209 & 17 & 36 \\
\hline $\begin{array}{l}\text { Multi-ring + } \\
\text { SADQ }\end{array}$ & 48.3 & 69.0 & 0 & 0 & 178,492 & 22,943 & 0 & 527 \\
\hline $\begin{array}{l}\text { Multi-ring + } \\
\text { Sub-queries }\end{array}$ & 398.8 & 120.8 & 0 & 0 & 624,837 & 57,518 & 0 & 230 \\
\hline $\begin{array}{l}\text { Partitioned-ring + } \\
\text { SADQ }\end{array}$ & 36.6 & 37.0 & 0 & 0 & 185,972 & 15,840 & 0 & 527 \\
\hline $\begin{array}{l}\text { Partitioned-ring + } \\
\text { Sub-queries }\end{array}$ & 40.7 & 16.4 & 0 & 0 & 432,859 & 46,946 & 0 & 527 \\
\hline $\begin{array}{l}\text { Overlapped-ring + } \\
\text { SADQ }\end{array}$ & 46.0 & 67.2 & 0 & 0 & 391,738 & 57,524 & 0 & 527 \\
\hline
\end{tabular}

The best design choice from each of the architectures is compared in Figure 14 The unstructured P2P-based architecture, which has the highest cost per query, is not shown to simplify the graph. The increase in advertising cost of centralized and superpeer architectures due to dynamic attributes is insignificant, as it is $O(1)$. Moreover, their query cost is independent of $a_{q},\left[l_{i}, u_{i}\right]$, and $m_{q}$. Therefore, they have the lowest cost per query. Cost of ring-based architectures tends to increase linearly. In Table X, we compare the three best ring-based designs with varying numbers of nodes $n$. Both the average cost per query and maximum query cost tend to increase linearly with $n$, confirming the analysis in Section 5.3. Therefore, query cost is bounded by $O(n)$. The cost of a partitioned ring-based architecture is lower as it is bounded by $O(n / A)$.

Table XI presents the query cost as well as per-node query load and index size. For comparison, according to Cai et al. [2004], we also generated range queries based on the uniform distribution of attributes and attribute values, where each attribute in a query specified $10 \%$ of the possible range of attribute values (i.e., $\left.\left(u_{i}-l_{i}\right)=0.1 D_{i}\right)$. Resource attributes were not changed. The performance of $\mathrm{RD}$ architectures is different when queries are formulated by selecting attributes and ranges of attribute values uniformly at random (irrespective of the actual resources). Not all random queries match resources in the system. Therefore, both the unstructured and superpeer architectures have to keep forwarding the queries until the $h_{T T L}$ expires. Consequently, the overall cost of RD increases. Furthermore, uniform queries cannot significantly benefit from $\mathrm{SADQ}$, as the minimum range of any attribute is always $10 \%$ of the domain, whereas in real queries (collected from SWORD), at least a few attributes tend to be somewhat specific (e.g., RespTime, 1MinLoad, and NumCores). Therefore, real-world queries can be resolved more efficiently using SADQ. Alternatively, given that the range of most of 


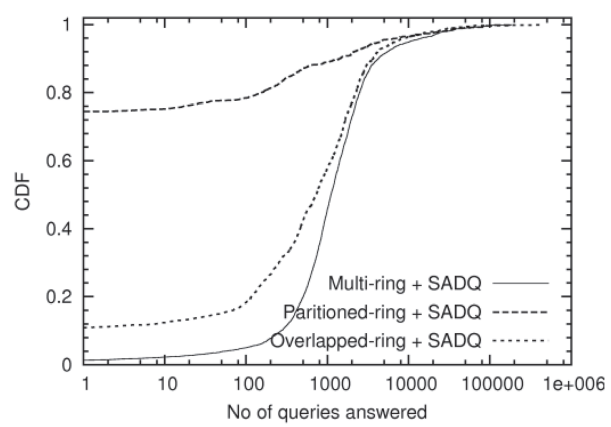

(a)

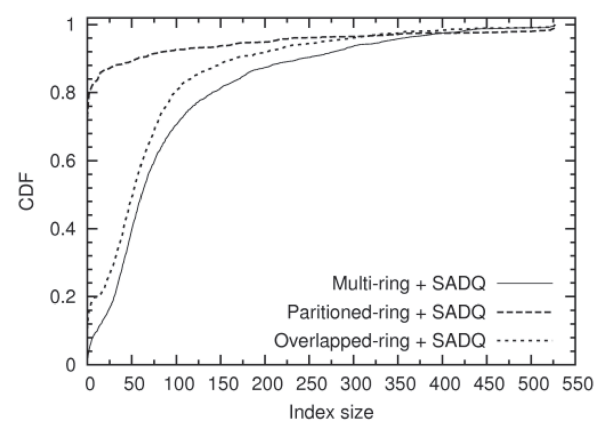

(b)

Fig. 15. Distribution of load: (a) Query load; (b) index size $(n=527)$.

the attribute values in real-world queries tend to be large, the aggregated query cost significantly increases when multiple subqueries are used.

The centralized solution had to index all the resources and answer all the queries issued within the system (950000 queries were issued during the simulation). Unstructured and superpeer architectures have a small index size, as either resources index themselves or superpeers index only a subset of the resources in the system. Furthermore, their query load is relatively balanced due to the use of random walks. Uniform queries are issued to different overlay rings/partitions with the same probability, hence distribute the query load among many nodes. Alternatively, due to the skewed distribution of attributes and attribute values in PlanetLab queries, a few rings/partitions and subset of the nodes within these rings/partitions are used to answer the majority of queries. This is the reason that the maximum number of SWORD queries answered by a node is 3.3-11.7 $\times$ higher than when answering uniform queries. The query load on a node that supports $\mathrm{SADQ}$ is relatively lower, as queries are resolved using the most selective attribute. Moreover, queries also terminate as soon as the desired number of resources is found. Hence, each node has to handle a relatively smaller number of queries. Figures 12 through 14 confirm that a partitioned ring with SADQ outperforms all other design choices. Figure 15(a) illustrates the query load distribution of architectures supporting SADQ. It can be seen that load distribution is skewed and a few nodes had to answer the majority of queries. It is particularly poor in the partitioned ring, where $74 \%$ of the nodes did not answer any query and one of the nodes answered $\sim 20 \%$ of all the queries. Such an imbalanced load distribution is not acceptable when the query rate is higher.

Because of the correlation and skewed distribution of attributes and attribute values, resources are not uniformly spread across the attribute space. Therefore, resources are indexed in only a small subset of the nodes in the ring. Multiple indexing used with SADQ and overlapped ring also force nodes to index many resources corresponding to different attributes. Moreover, some of the attributes have only a few valid attribute values and they are highly skewed, such as CPUArchi and NumCores. Such attribute values are indexed in few nodes and some of these nodes have to index a large fraction of the resources with the same attribute value. The index size distribution of solutions supporting SADQ is shown in Figure 15(b). In the partitioned ring, nodes that were mapped to less popular or unused partitions were never utilized. Therefore, it suffers significant load balancing issues, though it has the lowest RD cost among the structured P2P architectures. In conclusion, load balancing is a critical issue in all the designs (except in unstructured and superpeer designs), as the popularity of resources and queries is skewed and queries are less specific. 
Table XII. Design Space of Existing Solutions

\begin{tabular}{|c|c|c|c|c|c|c|c|}
\hline Architecture & \begin{tabular}{|c|} 
Advertising \\
Cost
\end{tabular} & $\begin{array}{l}\text { Query } \\
\text { Cost }\end{array}$ & $\begin{array}{c}\text { Query Load } \\
\text { Balanced }\end{array}$ & $\begin{array}{r}\text { Index Load } \\
\text { Balanced }\end{array}$ & $\begin{array}{c}\text { Many } \\
\text { Resources }\end{array}$ & $\begin{array}{c}\text { Many } \\
\text { Attribute }\end{array}$ & $\begin{array}{c}\text { Routing } \\
\text { State }\end{array}$ \\
\hline Centralized & Low & Low & No & No & No & Yes & Low \\
\hline Unstructured & None & High & Moderate & Yes & No & Yes & Low \\
\hline Superpeers & Low & Low & Moderate & Yes & Yes & Yes & Low \\
\hline $\begin{array}{l}\text { Multi-ring - } \\
\text { sub-queries }\end{array}$ & Moderate & High & No & No & No & No & High \\
\hline $\begin{array}{l}\text { Multi-ring - } \\
S A D Q\end{array}$ & High & Moderate & Moderate & No & No & No & High \\
\hline $\begin{array}{l}\text { Partitioned-ring - } \\
\text { sub-queries }\end{array}$ & Moderate & High & No & No & Moderate & Moderate & Low \\
\hline $\begin{array}{l}\text { Partitioned-ring - } \\
\text { SADQ }\end{array}$ & High & Moderate & Moderate & No & Moderate & Moderate & Low \\
\hline $\begin{array}{l}\text { Overlapped-ring - } \\
\text { SADQ }\end{array}$ & High & Moderate & No & No & Moderate & Moderate & Low \\
\hline$d$-Torus & None & High & No & Yes & Moderate & Moderate & Low \\
\hline
\end{tabular}

In summary, simulation-based analysis shows that real-world queries are relatively easier to resolve using unstructured, superpeer, and SADQ-based structured P2P architectures compared to uniform queries used in conventional studies. The cost of advertising dynamic attributes is significant and increases with $a_{q}$. While the query cost of ring-based DHTs is effectively $O(n)$, it does not increase with $a_{q}$. Moreover, except for unstructured and superpeer designs, all design choices are prone to significant load balancing issues where only a few nodes are involved in answering the majority of queries and indexing resources. Among the evaluated designs, the superpeer architecture has a good balance between the ability to find resources, overall cost of RD, and load balancing. These findings differ from Shen and Xu's [2012] analysis, which is based on conventional assumptions. For example, the authors concluded that the query cost is $O(\log n)$ and increases linearly with $a_{q}$. Moreover, they concluded that LORM could provide a superior index size distribution to other DHT-based solutions. Furthermore, the authors do not analyze the impact of dynamic attributes. Therefore, our analysis shows that existing design choices are unable to provide guaranteed RD while maintaining lower overhead and balanced load.

\section{IMPLICATIONS AND BEST PRACTICES FOR ENHANCING RESOURCE DISOVERY}

The design space of existing RD solutions is summarized in Table XII. From Tables IX and XII, it is apparent that none of the current design choices for RD simultaneously provides guaranteed $\mathrm{RD}$, efficiency, scalability, and load balancing under real workloads. The centralized architecture has the lowest cost per advertisement and query cost. A single message can be used to either advertise or query for a resource(s) irrespective of $a_{r}, a_{q},\left[l_{i}, u_{i}\right]$, and $m_{q}$. Lower advertising costs enables dynamic attributes to be advertised whenever their attribute values change while increasing the accuracy of indexed resources. As the central node is aware of all the resources in the system, it is also suitable for complex tasks such as matching multiple resources (for interresource bandwidth and latency), establishing a binding between a resource and an application that is interested in using it, and enforcing various incentives, trust, and security policies. However, a single node may not be able to handle all these messages. It further leads to a single point of failure and to privacy issues, as the central node can monitor usage patterns of resources. Nevertheless, centralized solutions are becoming more feasible, affordable, and reliable due to the recent advancements in distributed 
datacenter technologies. Therefore, when applicable, a centralized solution is still a desirable option.

The superpeer architecture is relatively efficient in resolving real-world queries that are less specific. Moreover, both the query load and index size are well balanced and independent of $a_{r}, a_{q}$, and $\left[l_{i}, u_{i}\right]$. Resources can afford to advertise dynamic attributes whenever they change, as the superpeer can be reached within one overlap hop. However, it does not provide a best-fit-type solution, where selected resources fit the minimum requirements of a query. Hence, applications that actually require a large number of high-capacity resources may not be able to find them, as they have been already allocated to applications with much lower resource demand. Trying to enforce best-fit-type matching will increase the query cost. Therefore, a superpeer architecture is more suitable for dynamic, best-effort environments such as mobile social and ad hoc networks. Due to high query cost and relatively low query hit rate, the unstructured $\mathrm{P} 2 \mathrm{P}$ architecture is not suitable for most applications. However, its high resilience to random node failures makes it suitable for highly dynamic environments such as small mobile social networks. Advertising agents are of little use as some dynamic attributes change very frequently, making the indexed resources stale. While it has been proposed to use aggressive timeouts to invalidate resources after a while, given the diversity in attributes and their rate of change, it is nontrivial to set a timeout.

While less specific queries do not affect the centralized architecture and increase the hit rate of the superpeer architecture, they increase the overhead of structured $\mathrm{P} 2 \mathrm{P}$ architectures. Though SADQ reduces the query cost, it significantly increases the advertising cost. Advertising cost is effectively doubled as old attribute values need to be removed from the rings to maintain consistency, as it is nontrivial to set a timeout. All the structured P2P architectures are prone to significant load balancing issues due to the small number of valid attribute values and their skewed distributions. Dynamic attributes are more important while predicting the performance of latency-sensitive applications (e.g., CASA and mobile social networks) and when resources are shared across multiple applications (CASA, GENI, grids, and clouds). Therefore, the applicability of $d$-torus is limited, as it cannot resolve those queries with only dynamic attributes. Though it seems most of the benefits of DHTs are lost under real workloads, they can still provide guaranteed RD, bounded performance, and are distributed. Moreover, as the locality of attribute values is preserved, they can also find those resources that fit the minimum requirements of a query. Hence, it becomes even more important to overcome their deficiencies to gain their benefits.

DHT designs typically assume that the domain of attributes is much larger than the number of nodes in the DHT (i.e., $D_{i} \gg n$ ) and that resources are uniformly spread within $D_{i}$. Therefore, it is preferable to add all the nodes to the DHT(s) expecting each node to index $\sim R / n$ resources. However, attributes such as CPUArchi, OS, and NumCores are not only skewed but also their domain sizes (i.e., number of distinct values in the domain) are much smaller (i.e., $D_{i} \ll n$ ). Even though the domain size of attributes such as CPUFree, 1MinLoad, DiskFree, and TxRate can be made arbitrarily large depending on the resolution, it is not useful to advertise them at a very high resolution, as users are not interested in finding very specific values. For example, advertising DiskFree at the granularity of a few megabytes is not suitable as $86 \%$ of the queries requested DiskFree values of 5-1000GB. Similarly, advertising TxRate at bps resolution is not useful as users are likely to query attribute ranges in tens to thousands of Kbps. Specifying attributes at a relatively low resolution is desirable (as far as it satisfies a query with the lowest $\left(u_{i}-l_{i}\right)$ ), as advertising cost can be reduced by ignoring minor changes in attribute values. It was observed that highly dynamic attributes that are queried using moderate to large ranges of attribute values contributed to more than $90 \%$ of the advertising cost in SADQ-based designs. Therefore, it is desirable to 
advertise attributes at a lower resolution (by applying a fixed or dynamic threshold) while reducing the effective domain sizes (i.e., $D_{i} \ll n$ ). Consequently, many nodes in the ring will not be able to index resources or answer queries. Moreover, adding all the nodes to a ring will unnecessarily increase the query cost, as both the average and worst-case query costs are proportional to $n$. Instead, in Bandara and Jayasumana [2012c], we show it is desirable to prune nodes that do not index any resources or answer any queries from a ring. However, this does not solve the problem of a few nodes having to index a large number of resources due to skewed distributions (e.g., when CPUArchi of $99 \%$ of the nodes are $\times 86$ ). One alternative is to append few random bits to a key (i.e., hash of an attribute value) such that identical resources will be mapped to different but adjacent nodes in the ring [Albrecht et al. 2008]. While this helps to distribute the index size better, it increases the query cost and does not balance the query load, as queries have to always start from the node corresponding to $l_{i}$. Bandara and Jayasumana [2012c] also present an alternative design where large index sets are split across multiple nodes that are added orthogonal to the ring. Adding nodes orthogonal to the rings does not increase the average and worst-case path length along the ring, hence the query cost is also reduced. We also used the same concept to balance the query load distribution. Another alternative is to explore hybrid designs where desirable properties such as lower advertising cost and load distribution in centralized and superpeer architectures are coupled with rings, for example, a ring of superpeers.

Performance of ring-based architectures can be approximated to $O(\log n)$ by using queries that are more specific. However, in practice, it is hard to determine specific resource requirements of an application. By specifying very specific queries, users also run the risk of not finding any useful resource. For example, a user may need only $500 \mathrm{MB}$ of disk space. If his/her query specified DiskFree $\in[500 \mathrm{MB}, 1000 \mathrm{MB}]$, the query is likely to fail as modern nodes have much greater free disk space. Therefore, users are compelled to specify a large range of attribute values.

The traces indicate that users tend to reissue queries with relaxed constraints once a query is unable to find the desired resources. For example, once a query for DiskFree $\in$ [500 MB, $1000 \mathrm{MB}]$ fails, a user may try DiskFree $\in[500 \mathrm{MB}, 10 \mathrm{~GB}]$. While this user-driven iterative querying approach leads to the discovery of needed resources, it increases the overhead both for the user and the system. Alternatively, a user may know only the total amount of required resources and not how they should be divided. For example, a user interested in getting (CPUCores $=4$ and MemSize $=4 \mathrm{~GB}$ ) may not care whether he/she receives two nodes, each with (CPUCores $=2$ and MemSize $=$ 2GB), or four nodes, each with (CPUCores $=1$ and MemSize $=1 \mathrm{~GB}$ ). Hence some queries may not specify $m_{q}$. An alternative solution for both these cases would be to allow for composite resources, where the system combines fine-grained resources into coarse-grained ones to meet the user requirements, such as by adapting approaches used within utility environments [Sahai et al. 2004]. While such optimizations usually belong to the resource matching phase [Bandara and Jayasumana 2012b], it is also possible to support identification or formation of such composite resources during the RD phase, for instance, by allowing a query (as it traverses the overlay) to keep track of potential resources that may be combined to meet the request. By exploring such optimizations, it may be possible to achieve better performance (e.g., $O(\log n)$ for a ring) and query success rate, if such complexities are incorporated into the RD solution, while enabling users to provide only the abstract details about their application requirements. Formation of composite resources by combining fine-grain resources in a flexible and efficient way within $\mathrm{P} 2 \mathrm{P}$ environments remains an open area to be investigated.

As the number of attributes increases, the performance of all the solutions degrades due to additional memory/storage requirements and increase in advertising cost. This 
is a concern in heterogeneous systems like CASA and GENI, both of which aggregate a multitude of diverse resources. One alternative is to represent multi-attribute resources using a few composite attributes. For example, BOINC uses a microbenchmark to rate nodes based on their integer and floating-point performance [Anderson and Reed 2009]. Cloud computing nodes are typically rated as high-memory, high-CPU, and cluster instances. A few attributes are attractive as they simplify and reduce the cost of RD. However, this method is not good at predicting the performance of arbitrary applications and is too abstract to be used in latency-sensitive applications such as CASA. Alternatively, applications that depend on more than one attribute cloud pick a minimum set of primary attributes that can accurately represent a resource. Dimension reduction techniques are also of interest. However, it would be challenging to resolve real-world queries that specify only a few attributes, as dimension reduction techniques are typically designed for those queries that specify all the attributes. Depending on the application(s) requirements, less informative attributes such as percentage of CPUFree and MemFree may be avoided.

Though many diverse applications run on the PlanetLab overlay, the structure of queries looked similar and requested only the computing resources. Hence, it was reasonable to use a representative mix of the queries together while conducting the performance analysis. However, vastly different applications with diverse resource requirements may run on systems like CASA and GENI. As discussed earlier, these systems combine significantly different resources such as radars and computing nodes. In such systems, the performance seen by different classes of applications could be significantly different due to the differences in query characteristics. Optimization of performance for a given mix of queries thus may not be effective, and evaluation of the system under such a mix may not be sufficient to understand its performance. More effective would be schemes that adapt to requirements of different classes of applications. A straightforward approach would be to have separate overlays for different types of resources with different parameters (e.g., with different resolutions and rate of advertising). However, this is not effective for collaborative P2P applications that rely on the ability to aggregate diverse resources. Such separation will increase their query cost while reducing the potential to aggregate the best set of resources. Therefore, it is important to develop better solutions that allow all the resources to be indexed in the same overlay but treated differently while resolving queries from different types of applications. In Bandara and Jayasumana [2013] we developed such a solution for single-attribute resources that allowed multiple communities of users with diverse interests to coexist in the same overlay but gain better query performance based on their specific interests. We wish to extend this solution to multiple attributes in the future. Generalized solutions need to be developed for handling different classes of applications in multi-attribute $\mathrm{P} 2 \mathrm{P}$ systems for a wide range of parameters and application classes.

As the existing solutions are applicable only under very specific scenarios, novel $\mathrm{RD}$ solutions are needed to overcome the performance and QoS issues posed by real workloads. Based on our findings, we recommend the following guidelines for designing novel RD solutions.

- Pick an architecture based on the requirements. A centralized architecture is for stable systems requiring high performance (independent of $a_{r}, a_{q},\left[l_{i}, u_{i}\right], m_{q}$, and $\lambda_{r}^{i}$ ), low overhead, resource matching, resource binding, and policy enforcement. A superpeer architecture is for dynamic/distributed systems requiring good performance (independent of $a_{r}, a_{q},\left[l_{i}, u_{i}\right], m_{q}$, and $\lambda_{r}^{i}$ ), moderate overhead, scalability, resource matching, and privacy. A single DHT with SADQ is recommended for 
distributed systems requiring guaranteed performance, moderate overhead, scalability, resource matching, and privacy.

- Support large $a_{r}$, few $a_{q}$, and large $\left[l_{i}, u_{i}\right]$. In most cases, allow users to specify simple queries with abstract details about application requirements, and let the $\mathrm{RD}$ system figure out which resources are a better fit. Use resource composition, key attributes, and/or dimension reduction to simplify advertising and querying. Support a diverse mix of applications and their requirements.

- Support dynamic attributes as they are popular and users are concerned about what resources are free. Consider the cost of advertising dynamic attributes and apply thresholds (static or dynamic) to reduce the advertising cost. If accurate representation of resources is required, explicitly remove previously advertised attribute values while advertising new ones.

- Balance the index size and query load distribution. Skewed distribution of resources and queries is common. Moreover, having highly skewed categorical attributes (e.g., CPUArchi) and applying thresholds to dynamic attributes reduce $D_{i}$. Therefore, the resources will be indexed in only a subset of the DHT nodes. Use small $D_{i}$ 's to improve query performance, such as in SADQ. Distribute large indexes and query loads to multiple nodes, for example, to multiple superpeers or by adding nodes orthogonal to a DHT ring. Therefore, based on the load, dynamically add/remove nodes to/from the DHT. Avoid adding random values to resource attributes in oder to distribute the load, as this increases query resolution cost.

- The ability to support other key phases of resource discovery, such as resource matching, binding, and composition, as well as security and privacy, is desirable.

- Hybrid approaches that combine the desirable features of centralized, superpeer, and ring-based architectures while taking into account the complex resource and query characteristics have the potential to provide better solutions.

- Evaluate the performance using real or synthetic traces that are derived using real-world resources and queries.

\section{CONCLUSIONS AND FUTURE WORK}

Fundamental design choices for P2P-based resource discovery were evaluated using the characteristics learned from four different real-world systems. Results presented demonstrated that real-world, multi-attribute resource and query characteristics diverge substantially from conventional assumptions. For example, while real-world, less specific queries are relatively easier to resolve than uniform queries, they introduce significant load balancing issues due to skewed resources and queries. Dynamic attributes contribute to high resource advertising cost, and their behavior is attributetype- and system specific, hence should not be ignored in performance studies. These findings indicate the need for more efficient, scalable, and robust resource discovery solutions as well as the importance of taking into account the specific characteristics of real-world resources and queries while designing and analyzing such solutions. Hybrid approaches that combine the desirable features of centralized, superpeer, and ring-based architectures have the potential to provide better solutions. We are working on a resource aggregation solution that can overcome current limitations while supporting resource matching, binding, and compensation.

\section{ACKNOWLEDGMENTS}

The authors thank V. Rudrappa for helping with data collection within our campus, Dr. J. Albrecht for providing the SWORD dataset, Dr. V.S. Pai for providing the CoMon dataset, and the Grid Observatory team for providing the grid dataset. 


\section{REFERENCES}

Albrecht, J., Oppenheimer, D., Vahdat, A., and Patterson, D. 2008. Design and implementation tradeoffs for wide-area resource discovery. ACM Trans. Internet Technol. 8, 4.

Anderson, D. P. and Fedak, G. 2006. The computational and storage potential of volunteer computing. In Proceedings of the 6th IEEE International Symposium on Cluster Computing and the Grid (CCGRID'06). 73-80.

Anderson, D. P. and Reed, K. 2009. Celebrating diversity in volunteer computing. In Proceedings of the 42 nd Hawaii International Conference on System Sciences (HICSS'09). 1-8.

Andrzejak, A., Kondo, D., and Anderson, D. P. 2010. Exploiting non-dedicated resources for cloud computing. In Proceedings of the 12th Network Operations and Management Symposium (NOMS'10). 341-348.

Bandara, H. M. N. D. 2012. Enhancing collaborative peer-to-peer systems using resource aggregation and caching: A multi-attribute resource and query aware approach. Ph.D. dissertation, Colorado State University, Fort Collins. http://hdl.handle.net/10217/78733.

Bandara, H. M. N. D. and Jayasumana, A. P. 2011a. On characteristics and modeling of P2P resources with correlated static and dynamic attributes. In Proceedings of the IEEE Global Telecommunications Conference (GLOBECOM'11). 1-6.

Bandara, H. M. N. D. and Jayasumana, A. P. 2011b. Characteristics of multi-attribute resources/queries and implications on P2P resource discovery. In Proceedings of the 9th ACS / IEEE International Conference on Computer Systems and Applications (AICCSA'11). 173-180.

Bandara, H. M. N. D. and Jayasumana, A. P. 2012a. Evaluation of P2P resource discovery architectures using real-life multi-attribute resource and query characteristics. In Proceedings of the IEEE Consumer Communications and Networking Conference (CCNC'12). 634-639.

Bandara, H. M. N. D. and Jayasumana, A. P. 2012b. Collaborative applications over peer-to-peer systemsChallenges and solutions. Peer-to-Peer Netw. Appl. 6, 3, 257-276.

Bandara, H. M. N. D. and Jayasumana, A. P. 2012c. Resource and query aware, peer-to-peer-based multiattribute resource discovery. In Proceedings of the 37th IEEE Conference on Local Computer Networks (LCN'12). 276-279.

Bandara, H. M. N. D. and Jayasumana, A. P. 2013. Community-based caching for enhanced lookup performance in P2P systems. IEEE Trans. Parallel Distrib. Syst. 24, 9, 1752-1762.

Bharambe, A. R., Agrawal, M., and Seshan, S. 2004. Mercury: Supporting scalable multi-attribute range queries. In Proceedings of the Conference on Applications, Technologies, Architectures, and Protocols for Computer Communications (SIGCOM'04). 353-366.

Briscoe, G. and Marinos, A. 2009. Digital ecosystems in the clouds: Towards community cloud computing. In Proceedings of the 3rd IEEE International Conference on Digital Ecosystems and Technologies (DEST'09). 103-108.

Brophy, J. and Bawden, D. 2005. Is Google enough? Comparison of an Internet search engine with academic library resources. Aslib Proc. 57, 6, 498-512.

Cai, M., Frank, M., Chen, J., and Szekely, P. 2004. MAAN: A multi-attribute addressable network for grid information services. J. Grid Comput. 2, 1, 3-14.

Costa, P., Napper, J., Pierre, G., and Van Steen, M. 2009. Autonomous resource selection for decentralized utility computing. In Proceedings of the 29th IEEE International Conference on Distributed Computing Systems (ICDCS'09). 561-570.

Elliott, C. 2009. GENI: Exploring networks of the future. http://www.geni.net.

George, M. 2006. B-A scale-free network generation and visualization. www.mathworks.com/matlabcentral/fileexchange/11947.

Germain-Renaud, C., Cady, A., Gauron, P., Jouvin, M., Loomis, C., et al. 2011a. The grid observatory. In Proceedings of the 11th IEEE/ACM International Symposium on Cluster, Cloud and Grid Computing (CCGRID'11). 114-123.

Germain-Renaud, C., Furst, F., Jouvin, M., Kassel, G., Nauroy, J., and Philippon, G. 2011b. The green computing observatory: A data curation approach for green IT. In Proceedings of the 9th IEEE International Conference on Dependable, Autonomic and Secure Computing (DASC'11). 798-799.

Heien, E. M., Kondo, D., and Anderson, D. P. 2012. A correlated resource models of Internet end hosts. IEEE Trans. Parallel Distrib. Syst. 23, 6, 977-984.

Ikeda, S., Kubo, I., and Yamashita, M. 2009. The hitting and cover times of random walks on finite graphs using local degree information. Theor. Comput. Sci. 410, 1, 94-100.

Iosup, A. and Epema, D. 2010. Grid computing workloads: Bags of tasks, workflows, pilots, and others. IEEE Internet Comput. 15, 2, 19-26. 
Kee, Y. S., Casanova, H., and Chien, A. 2004. Realistic modeling and synthesis of resources for computational grids. In Proceedings of the ACM/IEEE Conference on Supercomputing (SC'04).

Kim, W., Roopakalu, A., Li, K. Y., and Pai, V. S. 2011. Understanding and characterizing PlanetLab resource usage for federated network testbeds. In Proceedings of the Internet Measurement Conference (IMC'11).

Lazaro, D., Marques, J. M., Jorba, J., and Vilajosana, X. 2013. Decentralized resource discovery mechanisms for distributed computing in peer-to-peer environments. ACM Comput. Surv. 45, 4.

Lee, P., Jayasumana, A. P., Bandara, H. M. N. D., Lim, S., and Chandrasekar, V. 2012. A peer-to-peer collaboration framework for multi-sensor data fusion. J. Netw. Comput. Appl. 35, 3, 1052-1066.

Lu, D. and Dinda, P. A. 2003, Synthesizing realistic computational grids. In Proceedings of the ACM/IEEE Conference on Supercomputing (SC'03).

Lua, E. K., Crowcroft, J., Pias, M., Sharma, R., and Lim, S. 2004. A survey and comparison of peer-to-peer overlay network schemes. IEEE Comm. Surv. Tutor. 7, 2, 72-93.

Mclaughlin, D. and Chandrasekar, V. 2009. Short-wavelength technology and the potential for distributed networks of small radar systems. Bull. Amer. Meteorolog. Soc. 90, 1797-1817.

Newhouse, S. 2011. European grid infrastructure - An integrated sustainable Pan-European infrastructure for researchers in Europe (EGI-InSPIRE). Tech. rep. EGI-doc-201-v6. http://go.egi.eu/pdnon.

Park, K. and Pai, V. S. 2006. CoMon: A mostly-scalable monitoring system for PlanetLab. ACM SIGOPS Oper. Syst. Rev. 40, 1.

Sahai, A., Singhal, S., Machiraju, V., and Joshi, R. 2004. Automated policy-based resource construction in utility computing environments. In Proceedings of the Network Operations and Management Symposium (NOMS'04). 381-393.

Shen, H. 2009. A P2P-based intelligent resource discovery mechanism in Internet-based distributed Systems. J. Parallel Distrib. Comput. 69, 197-209.

Shen, H. and Hwang, K. 2012. Locality-preserving clustering and discovery of resources in wide-area distributed computational grids. IEEE Trans. Comput. 61, 4, 458-473.

Shen, H., Lin, Y., and Li, T. 2013. Combining efficiency, fidelity and flexibility in resource information services. IEEE Trans. Comput. 64, 2, 353-367.

Shen, H. and Xu, C.-Z. 2012. Leveraging a compound graph based DHT for multi-attribute range queries with performance analysis. IEEE Trans. Comput. 61, 4, 433-447.

Stoica, I., Morris, R., Liben-Nowell, D., Karger, D., Kaashoek, M. F., Dabek, F., and Balakrishnan, H. 2003. Chord: A scalable peer-to-peer protocol for Internet applications. IEEE/ACM Trans. Netw. 11, 1, 17-32.

Sulistio, A., Cibej, U., Venugopal, S., Robic, B., and Buyya, R. 2008. A toolkit for modelling and simulating data grids: An extension to Gridsim. Concurr. Comput. Pract. Exper. 20, 13, 1591-1609.

Tan, Y., Han, J., and Lu, Y. 2008. Agent-based intelligent resource discovery scheme in P2P networks. In Proceedings of the Pacific-Asia Workshop on Computational Intelligence and Industrial Application (PACIIA'08). 752-756.

Received December 2012; revised March 2014; accepted May 2014 